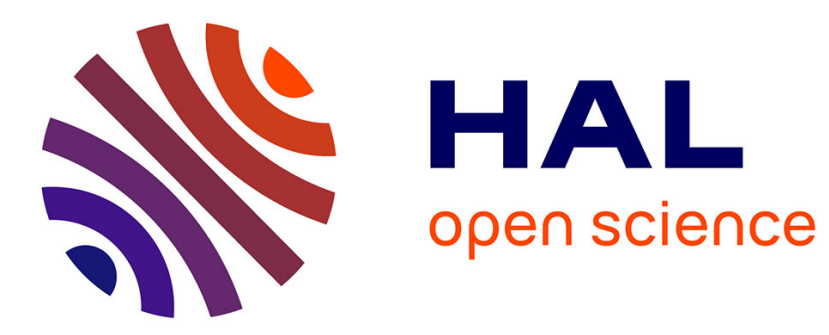

\title{
Variations sur un thème de Aldama et Shelah
}

Cédric Milliet

\section{To cite this version:}

Cédric Milliet. Variations sur un thème de Aldama et Shelah. The Journal of Symbolic Logic, 2016, 81 (1), pp.96-126. 10.1017/jsl.2014.67 . hal-00980379v2

\section{HAL Id: hal-00980379 \\ https://hal.science/hal-00980379v2}

Submitted on 15 Jan 2016

HAL is a multi-disciplinary open access archive for the deposit and dissemination of scientific research documents, whether they are published or not. The documents may come from teaching and research institutions in France or abroad, or from public or private research centers.
L'archive ouverte pluridisciplinaire HAL, est destinée au dépôt et à la diffusion de documents scientifiques de niveau recherche, publiés ou non, émanant des établissements d'enseignement et de recherche français ou étrangers, des laboratoires publics ou privés. 


\title{
VARIATIONS SUR UN THÈME DE ALDAMA ET SHELAH
}

\author{
CÉDRIC MILLIET
}

\begin{abstract}
We consider a group $G$ that does not have the independence property and study the definability of certain subgroups of $G$, using parameters from a fixed elementary extension $\mathbf{G}$ of $G$. If $\mathbf{X}$ is a definable subset of $\mathbf{G}$, its trace on $G$ is called an externally definable subset. If $\mathbf{H}$ is a definable subgroup of $\mathbf{G}$, we call its trace on $G$ an external subgroup. We show the following. For any subset $A$ of $G$ and any external subgroup $H$ of $G$, the centraliser of $A$, the $A$-core of $H$ and the iterated centres of $H$ are external subgroups. The normaliser of $H$ and the iterated centralisers of $A$ are externally definable. A soluble subgroup $S$ of derived length $\ell$ is contained in an $S$-invariant externally definable soluble subgroup of $G$ of derived length $\ell$. The subgroup $S$ is also contained in an externally definable subgroup $\mathbf{X} \cap G$ of $G$ such that $\mathbf{X}$ generates a soluble subgroup of $\mathbf{G}$ of derived length $\ell$. Analogue results are discussed when $G$ is merely a type definable group in a structure that does not have the independence property.
\end{abstract}

\section{Contents}

1. Preliminaries on the independence property 4

2. Preliminaries on NIP groups 8

3. External and discernible subgroups 13

$\begin{array}{ll}\text { 4. Envelopes in a definable group } & 17\end{array}$

5. Further chain conditions à la Baldwin Saxl 20

6. Envelopes in type definable groups 28

$\begin{array}{ll}\text { References } & 30\end{array}$

Given a group $G$, a subset $X \subset G$ is definable in $G$ if there exist a first-order formula $\varphi(x, \bar{y})$ and parameters $\bar{a}$ from $G$ such that $X$ consists of all $g \in G$ such that $\varphi(g, \bar{a})$ holds in $G$. A subset $X \subset G$ is externally definable if there is an elementary extension $\mathbf{G}$ of $G$ and parameters $\overline{\mathbf{a}}$ in $\mathbf{G}$ such that $X$ consists of all $g \in G$ such that $\varphi(g, \overline{\mathbf{a}})$ holds in $\mathbf{G}$. We write $\varphi(G, \overline{\mathbf{a}})$ for such a set $X$ if we want to stress on the defining formula $\varphi$, otherwise we write $\mathbf{X} \cap G$ where $\mathbf{X}$ stands for $\varphi(\mathbf{G}, \overline{\mathbf{a}})$. Definable subsets and externally definable ones coincide for the field $\mathbf{R}$ of real numbers (L. Van den Dries [vdD86]), for the field $\mathbf{Q}_{p}$ of $p$-adic numbers (F. Delon [Del89]), for an algebraically closed field and more generally for stable structures (it follows from the definability of types).

2010 Mathematics Subject Classification. 03C45, 03 C60.

Key words and phrases. Model theory; independence property; shattering type; VC-dimension; abelian, nilpotent, and soluble subgroups; nice subgroup; definable and type definable envelope.

Many thanks to the Referee for his careful readings and suggestions improving the clarity of the exposition. 
They do not coincide in general: in the ordered abelian group $(\mathbf{Q},+, \leqslant)$, the interval ]$\sqrt{2},+\infty[$ is not definable in $\mathbf{Q}$, but externally definable using the irrational parameter $\sqrt{2}$. Externally definable sets play an important role in structures that do not have the independence property, such as $(\mathbf{Q},+, \leqslant)$. They correspond to finite unions of convex subsets in the particular case of o-minimal and weakly o-minimal structures. Expanding the language of a weakly o-minimal structure by unary predicates interpreting finite unions of convex subsets preserves weak o-minimality (B. Baizhanov [Bai01]). Expanding the language of a structure that does not have the independence property by predicates interpreting externally definable subsets preserves the absence of the independence property (S. Shelah [She09]).

A group $G$ does not have the independence property if for every first order formula $\varphi(x, \bar{y})$, the Vapnik-Chervonenkis dimension of the family $\{\varphi(G, \bar{g}): \bar{g} \in G\}$ is finite. We shall also use the short hand NIP group. S. Shelah [She09] and R. de Aldama [dA13] began investigating definable subgroups of $G$ using external parameters lying in a fixed $|G|^{+}$-saturated elementary extension $\mathbf{G}$ of $G$. S. Shelah showed that if $G$ has an infinite abelian subgroup $A$, there exists a definable abelian subgroup of $\mathbf{G}$ that contains infinitely many elements of $A$. R. de Aldama went on showing that for any nilpotent subgroup $N \subset G$, there is a definable nilpotent subgroup of $\mathbf{G}$ that contains $N$ and has the same nilpotency class as $N$, and that for any soluble subgroup $S \subset G$ that is normal in $\mathbf{G}$, there is a definable soluble subgroup of $\mathbf{G}$ that contains $S$ and has the same derived length as $S$. As we were further investigating the soluble case trying to get rid of the strong normality assumption, we had to cope with subgroups closely related to the infinitesimal numbers, in the following way: in a non-principal ultrapower $\mathbf{R}^{\mathcal{U}}$ of the field of real numbers, the subgroup $\mathfrak{I}$ of infinitesimal numbers is not definable in $\mathbf{R}^{\mathcal{U}}$. There is an external parameter $\varepsilon$ in an elementary extension $\mathfrak{R}$ of $\mathbf{R}^{\mathcal{U}}$ such that $\mathfrak{I}=]-\varepsilon, \varepsilon[$, so that $\mathfrak{I}$ is externally definable as a set. $\mathfrak{I}$ is not the trace of a definable subgroup of $\mathfrak{R}$, however it is the conjunction of the uniform filtering family of symmetric definable sets $] \frac{-1}{n}, \frac{1}{n}\left[\right.$ that defines a group both in $\mathbf{R}^{\mathcal{U}}$ and $\mathfrak{R}$.

We call a subgroup $H \subset G$ discernible if there is a subgroup $\mathbf{H} \subset \mathbf{G}$ that is the intersection of a uniform filtering family of symmetric definable subsets of $\mathbf{G}$ such that $H=\mathbf{H} \cap G$ (we call $\mathbf{H}$ a nice subgroup of $\mathbf{G}$ ). Discernible subgroups are examples of externally definable subsets, and in the particular case when $G$ is a stable group, they coincide with definable subgroups (see Lemma 3.6). We call the subgroup $H \subset G$ external if there is a definable subgroup of $\mathbf{H} \subset \mathbf{G}$ such that $H=\mathbf{H} \cap G$. Our main results are the following.

Theorem 0.1 (finding external subgroups). Let $G$ be a NIP group, G a $|G|^{+}$-saturated elementary extension of $G$ and $H=\mathbf{H} \cap G$ an external subgroup of $G$.

(1) There is $n \in \omega$ such that for every $A \subset G$, there are $\mathbf{a}_{1}, \ldots, \mathbf{a}_{n}$ in $\mathbf{G}$ such that

$$
C_{G}(A)=C_{G}\left(\mathbf{a}_{1}, \ldots, \mathbf{a}_{n}\right) .
$$

(2) For every $n \in \omega$, there is a definable subgroup $\mathbf{K} \subset \mathbf{G}$ such that

$$
H=\mathbf{K} \cap G \quad \text { and } \quad Z_{n}(H)=Z_{n}(\mathbf{K}) \cap G .
$$

(3) There is $n \in \omega$ such that for every $A \subset G$, there are $\mathbf{a}_{1}, \ldots, \mathbf{a}_{n}$ in $\mathbf{G}$ such that

$$
\bigcap_{a \in A} H^{a}=\mathbf{H}^{\mathbf{a}_{1}} \cap \cdots \cap \mathbf{H}^{\mathbf{a}_{n}} \cap G .
$$


Theorem 0.2 (finding discernible subgroups). Let $G$ be a NIP group, G a $|G|^{+}$-saturated, $|G|^{+}$-homogeneous elementary extension of $G$ and $H=\mathbf{H} \cap G$ a discernible subgroup of $G$.

(1) For every $n \in \omega$, there are nice subgroups $\mathbf{K}, \mathbf{Z}_{n} \subset \mathbf{G}$ such that

$$
H=\mathbf{K} \cap G, \quad Z_{n}(H)=\mathbf{Z}_{n} \cap G \quad \text { and } \quad \mathbf{Z}_{n} \subset Z_{n}(\mathbf{K}) .
$$

(2) There are $n \in \omega$ and a nice subgroup $\mathbf{K} \subset \mathbf{G}$ such that for every $A \subset G$, there are $\mathbf{a}_{1}, \ldots, \mathbf{a}_{n}$ in $\mathbf{G}$ with

$$
H=\mathbf{K} \cap G \quad \text { and } \quad \bigcap_{a \in A} H=\mathbf{K}^{\mathbf{a}_{1}} \cap \cdots \cap \mathbf{K}^{\mathbf{a}_{n}} \cap G .
$$

(3) There are nice subgroups $\mathbf{K} \subset \mathbf{H}$ and $\mathbf{N} \subset N_{\mathbf{G}}(\mathbf{K})$ of $\mathbf{G}$ such that

$$
H=\mathbf{K} \cap G \quad \text { and } \quad N_{G}(H)=\mathbf{N} \cap G .
$$

(4) There are $n \in \omega$ and a nice subgroup $\mathbf{K} \subset \mathbf{G}$ such that for every $A \subset G$ and $\mathfrak{A} \subset \operatorname{Aut}(G / A)$, there are $\boldsymbol{\sigma}_{1}, \ldots, \boldsymbol{\sigma}_{n}$ in $\operatorname{Aut}(\mathbf{G} / A)$ with

$$
H=\mathbf{K} \cap G \quad \text { and } \quad \bigcap_{\sigma \in \mathfrak{A}} H^{\sigma}=\mathbf{K}^{\sigma_{1}} \cap \cdots \cap \mathbf{K}^{\sigma_{n}} \cap G .
$$

(5) For every $n \in \omega$ and $A \subset G$, the nth-centraliser $C_{G}^{n}(A)$ is a discernible subgroup of $G$.

Theorem 0.3 (soluble envelopes). Let $G$ be a NIP group, $S \subset G$ a soluble subgroup of derived length $\ell$ and $\mathbf{G} a|G|^{+}$-saturated elementary extension of $G$.

(1) There is a nice subgroup $\mathbf{H} \subset \mathbf{G}$ with $S \subset \mathbf{H}$ such that $\mathbf{H}$ is soluble of derived length $\ell$, $\mathbf{H} \cap G$ is $S$-invariant and normalised by $N_{G}(S)$.

(2) There is a definable subset $\mathbf{X} \subset \mathbf{G}$ with $S \subset \mathbf{X}$ such that $\mathbf{X} \cap G$ is a subgroup of $G$ and $\mathbf{X}$ generates a soluble subgroup of $\mathbf{G}$ of derived length $\ell$.

(3) If $S$ is in addition normal in $G$, there is a normal, soluble of derived length $\ell$, definable subgroup $\mathbf{H} \subset \mathbf{G}$ with $S \subset \mathbf{H}$.

NIP groups include finite groups, abelian groups in the pure language of groups (W. Szmielew [Szm55]), abelian ordered groups (Y. Gurevich and P. Schmitt [GS84]), groups definable in a stable structure (e.g. linear algebraic groups over separably closed fields, C. Wood [Woo79]) and groups definable in an o-minimal structure (e.g. linear algebraic groups over the field of real numbers). These are trivial ones for most of the considerations of this paper, as both stable and o-minimal groups satisfy strong descending chain conditions, either on uniformly definable subgroups [BS76] or on all definable subgroups [Pil88]: in such a group $G$, for every $A \subset G$, the centraliser $C_{G}(A)$ and the $A$-core $\bigcap_{a \in A} a^{-1} H a$ of a definable subgroup $H \subset G$ are definable, and these properties remain true in quotients of $G$ by normal definable subgroups. Other examples include linear algebraic groups over a field $k$ that does not have the independence property, and more generally groups interpretable therein, e.g. quotients $H_{1} / H_{2}$ where $H_{2} \triangleleft H_{1}$ are definable subgroups (not necessarily Zariski-closed) of the general linear group $\mathrm{GL}_{\mathrm{n}}(k)$ in a field structure $(k, L)$ where $L$ is an expansion of the field language such that the structure $(k, L)$ is NIP. This holds in particular with $k$ equal to (a finite algebraic extension of) the pure field $\mathbf{Q}_{p}$ of $p$-adic numbers (L. Matthews [Mat93], see also [Bél12]) and more generally to a Henselian valued field of characteristic 0 whose residue field is NIP (F. Delon 
[Del81]). Other examples of NIP fields: the valued field $\bigcup_{n \geqslant 1} \mathbf{F}_{p}^{a l g}\left(\left(t^{1 / n}\right)\right)$ of Puisieux series over $\mathbf{F}_{p}^{a l g}$ and more generally any valued field of charactersitic $p>0$ with perfect NIP residue field, with $p$-divisible value group and with no proper algebraic valuated extension having ramification index 1 and residue degree 1 (I. Kaplan, T. Scanlon and F. Wagner [KSW11]). In a linear algebraic group $\mathrm{G}(k)$ over a field $k$, every descending chain of Zariski-closed subgroups has finite length. In particular, for any subset $A \subset G(k)$, the centraliser $C_{G(k)}(A)$ is definable, and the $A$-core of a Zariski-closed subgroup $H \subset G(k)$ is Zariski-closed (hence definable), but the $A$-core of a definable subgroup may not be definable.

NIP groups also include general linear groups $\mathrm{GL}_{\mathrm{n}}(R)$ over a NIP ring $R$, which may be a domain (such as valuation rings of the valued fields cited above) or not (such as any nonprincipal ultraproduct $\prod_{\mathcal{U}} \mathbf{Z} / p^{n} \mathbf{Z}$ for a fixed prime number $p$ ).

Two examples of a less algebraic nature. The universal covering group $\widetilde{G}$ of a definably connected group $G$ that is interpretable in an o-minimal expansion $M$ of the field $\mathbf{R}$ is a NIP group: $\widetilde{G}$ is interpretable in the two sorted structure $\left(\left(\pi_{1}(\mathrm{G}),+\right), M\right)$ (E. Hrushovski et al. [HPP11]) hence NIP (A. Conversano and A. Pillay [CP12]). An ultraproduct of groups that are uniformly interpretable in a NIP structure is NIP (D. Macpherson and K. Tent [MT12]).

\section{Preliminaries ON THE INDEPENDENCE PROPERTY}

Before discussing the particular case of groups, we consider an arbitrary first-order language $L$, a complete theory $T$, one of its models $M$ and a subset $A \subset M$. Let $\bar{x}$ and $\bar{y}$ be disjoint tuples of variables of respective length $p \geqslant 1$ and $q \geqslant 1$. Given a formula $\phi(\bar{x})$ and a partial type $\rho(\bar{x})$ with parameters in $M$, i.e. a set of formulas consistent with the $L \cup M$-theory of $M$, we write $\phi(A)$ for the subset $\left\{\left(x_{1}, \ldots, x_{p}\right) \in A^{p}: \phi\left(x_{1}, \ldots, x_{p}\right)\right.$ holds in $\left.M\right\}$ of $M^{p}$ and $\rho(A)$ for the intersection $\bigcap_{\phi \in \rho} \phi(A)$.

1.1. Shattering formulas. Let $\varphi(\bar{x}, \bar{y})$ be a formula in $p+q$ variables with possible parameters in $M$. Given a non-zero $n \in \omega$, we say that the formula $\varphi(\bar{x}, \bar{y})$ shatters $n$ in $T$ if there are parameters $\bar{a}_{1}, \ldots, \bar{a}_{n}$ in $M^{p}$ and $\left(\bar{b}_{J}\right)_{J \subset\{1, \ldots, n\}}$ in $M^{q}$ such that

$$
\left(M \models \varphi\left(\bar{a}_{i}, \bar{b}_{J}\right)\right) \Longleftrightarrow i \in J
$$

In other words, $\varphi(\bar{x}, \bar{y})$ shatters $n$ in $T$ if there is a finite subset $A \subset M^{p}$ with $n$ elements whose subsets are all of the form $A \cap \varphi(M, \bar{b})$ for some $\bar{b}$ varying in $M^{q}$. As shattering $n$ is a first order property, it does not depend on the model $M$ of $T$ chosen. We call VapnikChervonenkis dimension of $\varphi(\bar{x}, \bar{y})$ in $T$, sometimes omitting to specify $T$ when the ambient theory is obvious, the maximal $n \in \omega$ that is shattered by $\varphi(\bar{x}, \bar{y})$ in $T$ if such a number exists, or $\infty$ otherwise. We write it $V C(\varphi)$. Note that $V C(\varphi)$ equals $V C(\neg \varphi)$. In these definitions, the tuples of variables $\bar{x}$ and $\bar{y}$ do not play the same role. We write $\varphi^{*}(\bar{x}, \bar{y})$ for the dual formula of $\varphi(\bar{x}, \bar{y})$, obtained by interchanging the role of $\bar{x}$ and $\bar{y}$. We say that $\varphi(\bar{x}, \bar{y})$ has the independence property in $T$, if it $^{1}$ has infinite VC-dimension in $T$. The structure $M$ or

\footnotetext{
${ }^{1}$ According to Shelah's definition in [She90, Definition 4.2], $\varphi(\bar{x}, \bar{y})$ has the independence property if the dual formula $\varphi^{*}(\bar{x}, \bar{y})$ has infinite VC-dimension. The two statements are equivalent as $\operatorname{VC}(\varphi) \leqslant n$ implies $V C\left(\varphi^{*}\right) \leqslant 2^{n}$ by [Poi85, Lemme 12.16].
} 
its theory $T$ do not have the independence property (i.e. are NIP) if no formula has the independence property in $T$, i.e. if every formula has a finite VC-dimension.

The relation between Shelah's independence property in [She90] and Vapnik-Chervonenkis' dimension in [VC71] is pointed out in [Las92]. We refer to [She90] and [Adl14] for more about NIP structures.

1.2. Shattering types. We extend the previous definitions to partial types. Let $\lambda$ and $\mu$ be two cardinal numbers, with $\mu \leqslant \lambda^{+}$. If $\pi(\bar{x}, \bar{y})$ is a partial type in $p+q$ variables, we say that $\pi(\bar{x}, \bar{y})$ shatters $\lambda$ up to $\mu$ in $T$ if there is an elementary extension $\mathbf{M}$ of $M$, a subset $A \subset \mathbf{M}^{p}$ with $|A|=\lambda$ such that for every $B \subset A$ with $|B|<\mu$ there is $\bar{b} \in \mathbf{M}^{q}$ such that $B=A \cap \pi(\mathbf{M}, \bar{b})$. Equivalently, there is an elementary extension $\mathbf{M}$ of $M$, a family $\left\{\bar{a}_{i}: i<\lambda\right\}$ of elements of $\mathbf{M}^{p}$, and a family $\left\{\bar{b}_{J}: J \subset \lambda\right.$, and $\left.|J|<\mu\right\}$ of elements of $\mathbf{M}^{q}$ such that

$$
\left(\mathbf{M} \models \pi\left(\bar{a}_{i}, \bar{b}_{J}\right)\right) \Longleftrightarrow i \in J
$$

We say that $\pi(\bar{x}, \bar{y})$ co-shatters $\lambda$ in $T$ up to $\mu$ if there is an elementary extension $\mathbf{M}$ of $M$ and families $\left\{\bar{a}_{i}: i<\lambda\right\}$ and $\left\{\bar{b}_{J}: J \subset \lambda\right.$, and $\left.|J|<\mu\right\}$ whose elements lie respectively in $\mathbf{M}^{p}$ and $\mathbf{M}^{q}$, such that

$$
\left(\mathbf{M} \models \pi\left(\bar{a}_{i}, \bar{b}_{J}\right)\right) \Longleftrightarrow i \notin J
$$

or equivalently if there is $A \subset \mathbf{M}^{p}$ of cardinal $\lambda$ such that for all $B \subset A$ with $|A \backslash B|<\mu$ there is $\bar{b} \in \mathbf{M}^{q}$ with $B=A \cap \pi(\mathbf{M}, \bar{b})$.

If $\pi(\bar{x}, \bar{y})$ shatters $\lambda$ up to $\lambda^{+}$, then it shatters and co-shatters $\lambda$ up to every $\mu \leqslant \lambda^{+}$. In this case, we simply say that $\pi(\bar{x}, \bar{y})$ shatters $\lambda$.

If a formula $\varphi(\bar{x}, \bar{y})$ shatters every $n \in \omega$ in $T$, by the Compactness Theorem, $\varphi(\bar{x}, \bar{y})$ shatters $\lambda$ for every cardinal number $\lambda$. If a partial type $\pi(\bar{x}, \bar{y})$ shatters every $n \in \omega$ in $T$, by the Compactness Theorem, for every $n \in \omega$, there is a finite conjunction of formulas in $\pi(\bar{x}, \bar{y})$ that has VC-dimension at least $n$. However, it is possible that $T$ be NIP.

Example 1.1 (The Cantor ternary set in $\mathbf{R}$ ). The Cantor ternary set $\mathfrak{C}$ is the intersection of the closed sets $C_{n}$ defined by

$$
C_{0}=[0,1] \text { and } C_{n+1}=\frac{C_{n}}{3} \cup\left(\frac{2}{3}+\frac{C_{n}}{3}\right) .
$$

$\mathfrak{C}$ consists of the elements of $[0,1]$ having at least one ternary representation whose digits belong to $\{0,2\}$. The partial type $\left\{x+y \in C_{n}: n \in \omega\right\}$ shatters $\omega$. For every $i \in \omega$ and subset $J \subset \omega$, we define

$$
a_{i}=\left(\frac{1}{3}+\frac{1}{3^{2}}\right) \times \frac{1}{3^{2 i}} \quad \text { and } \quad b_{J}=\sum_{j \in J} \frac{2}{3^{2 j+2}},
$$

so that we have

$$
a_{i}+b_{J}=\frac{1}{3^{2 i+1}}+\frac{1}{3^{2 i+2}}+\sum_{j \in J} \frac{2}{3^{2 j+2}} .
$$

On the one hand, if $i \in \omega \backslash J$, then $a_{i}+b_{J}$ has occurrences of 1 in every ternary representation. On the other hand, if $i \in J$, then

$$
a_{i}+b_{J}=\frac{2}{3^{2 i+1}}+\sum_{j \in J \backslash\{i\}} \frac{2}{3^{2 j+2}} .
$$


Example 1.2 (The Cantor ternary set in $\mathbf{Q}_{3}$ ). The 3-adic ternary Cantor set $\mathfrak{C}_{3}$ is the intersection of the closed subsets $C_{n} \subset \mathrm{Q}_{3}$ defined by

$$
C_{0}=\mathbf{Z}_{3} \quad \text { and } \quad C_{n+1}=3 C_{n} \cup\left(2+3 C_{n}\right) .
$$

$\mathfrak{C}_{3}$ consists of the 3 -adic integers whose canonical expansion have coefficients in $\{0,2\}$ (M. Lapidus and H. Lũ [LL08]). The ring of 3-adic integers is defined by the formula $(\exists y)\left(y^{2}=\right.$ $\left.1+3 x^{2}\right)$ and $\mathfrak{C}_{3}$ is a type definable subset of $\mathbf{Q}_{3}$. The type $x+y \in \mathfrak{C}_{3}$ shatters $\omega$ in $\mathbf{Q}_{3}$, which can be seen taking $a_{i}=3^{2 i}+3^{2 i+1}$ and $b_{J}=\sum_{j \in J} 2 \cdot 3^{2 j}$. Note that the field $\mathbf{Q}_{3}$ does not have the independence property by [Mat93] (see also [Bél12, Section 4.2]).

Given a cardinal $\lambda$, here is an example of a language $L_{\lambda}$, an $L_{\lambda}$-structure $M$ and partial type $\pi_{\lambda}(x, y)$ that shatters $\lambda$ in $M$. Let $\Gamma$ be an ordered abelian divisible group containing a copy of $\lambda$. Consider the Hahn field $\mathbf{Q}_{3}((\Gamma))$ of generalised power series with 3-adic coefficients. Consider the structure $\left(\mathbf{Q}_{3}((\Gamma)),+, 0, P_{n}^{\mu}: \mu \in \lambda, n \in \omega\right)$ where $P_{n}^{\mu}$ is a unary predicate interpreting the subgroup of $\mathbf{Q}_{3}((\Gamma))$ whose elements are of the form $\sum_{i \in I} a_{i} t^{i}$ with $a_{\mu} \in$ $3^{n} \mathbf{Z}_{3}$. In this language, $\mathbf{Q}_{3}((\Gamma))$ is an abelian structure in the sense of E. Fisher (see [Fis77], or [Wag97, Example 0.3.1]). Any definable subset of the Cartesian product $\mathbf{Q}_{3}((\Gamma))^{n}$ is a Boolean combination of cosets of $\operatorname{acl}(\emptyset)$-definable subgroups of $\mathbf{Q}_{3}((\Gamma))^{n}$ by [Wei93] (see also [Wag97, Theorem 4.2.8]): the structure $\left(\mathrm{Q}_{3}((\Gamma)),+, 0, P_{n}^{\mu}: \mu \in \lambda, n \in \omega\right)$ is stable.

Let $\mathfrak{C}_{3}^{\lambda}$ denote the partial type defined by

$$
\mathfrak{C}_{3}^{\lambda}=\bigcap_{\mu \in \lambda} \bigcap_{n \in \omega} C_{n}^{\mu}, \quad \text { where } \quad C_{0}^{\mu}=P_{0}^{\mu} \quad \text { and } \quad C_{n+1}^{\mu}=3 C_{n}^{\mu} \cup\left(2+3 C_{n}^{\mu}\right) .
$$

The realisations of $\mathfrak{C}_{3}^{\lambda}$ in $\mathbf{Q}_{3}((\Gamma))$ are the elements of the form $\sum_{i \in I} a_{i} t^{i}$ where $a_{\mu} \in \mathfrak{C}_{3}$ for each cardinal number $\mu<\lambda$ belonging to $I$. The families $a_{i}=4 t^{i}$ and $b_{J}=\sum_{j \in J} 2 t^{j}$ witness that the type $x+y \in \mathfrak{C}_{3}^{\lambda}$ shatters $\lambda$ in $\mathbf{Q}_{3}((\Gamma))$.

Example 1.3 (A type that shatters every $n \in \omega$, but not $\omega$ up to 4 ). In $(\mathbf{R},+, \leqslant)$, here is a sequence of definable subsets $A_{n} \subset[0,1]$ such that the partial type $\pi(x, y)=\left\{x-y \in A_{n}\right.$ : $n \in \omega\}$ shatters every $n \in \omega$ but does not shatter $\omega$ up to 4 . Define for all $n \in \omega$, a definable subset $B_{n} \subset[n, n+1]$ of the form

$$
\left.B_{n}=\right] n, n+1\left[\backslash\left\{c_{n, 1}, \ldots, c_{n, 2^{n}}\right\} \quad \text { with } n<c_{n, 1}<\cdots<c_{n, 2^{n}}<n+1\right.
$$

such that, for all $i \in\{1, \ldots, n\}$ and $J \subset\{1, \ldots, n\}$ there are $a_{n, i} \in \mathbf{R}$ and $b_{n, J} \in \mathbf{R}$ such that $\left.\left(a_{n, i}-b_{n, J}\right) \in\right] n, n+1[$ and

$$
\left(a_{n, i}-b_{n, J}\right) \in B_{n} \Longleftrightarrow i \in J .
$$

We put $C=\left\{c_{i, j}: i, j \in \omega\right\}$, and we may build each $B_{n}$ so that the map mapping a 2 element subset $\{x, y\}$ of $C$ to $|x-y|$ has finite (unbounded) fibres (using a $\mathbf{Q}$-basis of $\mathbf{R}$ for instance). We put for all $n \in \omega$,

$$
\left.\left.A_{n}=\right]-\infty, 0\right] \cup B_{0} \cup B_{1} \cup \cdots \cup B_{n} \cup[n+1,+\infty[
$$

It follows that, for every $n \in \omega$ and finite $J \subset \omega$,

$$
\left(\mathbf{R}=\pi\left(a_{n, i}, b_{n, J}\right)\right) \Longleftrightarrow i \in J,
$$


so $\pi(x, y)$ shatters every $n \in \omega$. If $X$ is an infinite set shattered by $\pi(x, y)$, then there is a real number $\ell$ and infinitely many 3 elements subsets $Y$ of $X$ with the property that $|x-y|=\ell$ for some $x$ and $y$ in $Y$. This shows that $\pi(x, y)$ does not shatter $\omega$ up to 4 .

1.3. Nice sets. The previous examples show that shattering types can occur in a NIP theory. We go on by giving one elementary condition under which a shattering type yields a formula with the independence property.

A partial type $\rho(\bar{x})$ with parameters in $M$ is uniform if there is a formula $\varphi(\bar{x}, \bar{y})$ and a subset $A \subset M^{q}$ such that

$$
\rho(\bar{x})=\{\varphi(\bar{x}, \bar{a}): \bar{a} \in A\} .
$$

A family $\mathfrak{F}$ of subsets of $M^{p}$ is uniformly definable if there is a uniform $p$-type $\rho(\bar{x})$, such that

$$
\mathfrak{F}=\{\phi(M): \phi \in \rho\} .
$$

$\mathfrak{F}$ is a filter if for every $X$ and $Y$ in $\mathfrak{F}$, there is $Z$ in $\mathfrak{F}$ such that $Z \subset X \cap Y$. We say that $\rho(\bar{x})$ is a filter if the family $\{\phi(M): \phi \in \rho\}$ is a filter, in other words if for every $\phi_{1}$ and $\phi_{2}$ in $\rho$, there exists $\phi$ in $\rho$ such that

$$
\rho \models \forall \bar{x}\left(\phi(\bar{x}) \rightarrow \phi_{1}(\bar{x}) \wedge \phi_{2}(\bar{x})\right) .
$$

Definition 1.4 (nice subset). A partial type $\rho(\bar{x})$ with parameters in $M$ is nice if $\rho(\bar{x})$ is a uniform filter. A subset $X \subset M^{p}$ is nice (in $M$ ) if there is a nice partial type $\rho(\bar{x})$ such that $X=\rho(M)$.

Definition 1.5 (externally definable subset). A subset $X \subset M^{n}$ is externally definable if there is an elementary extension $\mathbf{M}$ of $M$ and a definable subset $\mathbf{X} \subset \mathbf{M}^{n}$ such that $X=\mathbf{X} \cap M^{n}$. Equivalently, there is a formula $\varphi(\bar{x}, \bar{y})$ and a tuple $\mathbf{c}$ in $\mathbf{M}$ such that

$$
X=\left\{\bar{x} \in M^{n}: \mathbf{M} \models \varphi(\bar{x}, \mathbf{c})\right\} .
$$

Lemma 1.6. A nice set is externally definable.

Proof. Let $X=\rho(M)$ with $\rho(\bar{x})=\{\varphi(\bar{x}, \bar{a}): \bar{a} \in A\}$ a uniform filter. The partial type

$$
\pi(\bar{y})=\{\varphi(\bar{b}, \bar{y}), \forall \bar{x}(\varphi(\bar{x}, \bar{y}) \rightarrow \varphi(\bar{x}, \bar{a})): \bar{b} \in X, \bar{a} \in A)\}
$$

is finitely satisfiable in $A$. It follows that $X=\varphi(M, \overline{\mathbf{a}})$ for any realisation $\overline{\mathbf{a}}$ of $\pi$.

Corollary 1.7. If there is a nice partial type that shatters every natural number $n$ in $T$, then $T$ has the independence property.

Proof. If $\pi(\bar{x}, \bar{y})=\{\varphi(\bar{x}, \bar{y}, \bar{a}): \bar{a} \in A\}$ is a nice partial type that shatters every $n$, there are an elementary extension $\mathbf{M}_{1}$ of $M$ and tuples $\left\{\bar{a}_{i}^{n}: i<n\right\},\left\{\bar{b}_{J}^{n}: J \subset n\right\}$ in $\mathbf{M}_{1}$ such that

$$
\left(\mathbf{M}_{1} \models \pi\left(\bar{a}_{i}^{n}, \bar{a}_{J}^{n}\right)\right) \Longleftrightarrow i \in J
$$

for all $n$. By Lemma 1.6, there are an elementary extension $\mathbf{M}_{2}$ of $\mathbf{M}_{1}$ and $\overline{\mathbf{a}} \in \mathbf{M}_{2}$ such that $\pi\left(\mathbf{M}_{1}\right)=\varphi\left(\mathbf{M}_{1}, \overline{\mathbf{a}}\right)$, so $\varphi(\bar{x}, \bar{y}, \overline{\mathbf{a}})$ has the independence property in $\mathbf{M}_{2}$.

Remark 1.8. In Corollary 1.7, one can neither drop the assumption that the partial type is uniformly definable, nor drop the assumption that the partial type is a filter: the type of Example 1.3 is equivalent to a uniform type, to a filter also, but not to a uniform filter. 
Corollary 1.9. Let $M$ be a NIP L-structure, with = being the only relation symbol in $L$. Let $N \subset M$ and $E \subset N^{2}$ be nice subsets (in $M$ ) such that $N$ is a substructure of $M$ and $E$ is an equivalence relation on $N$ that preserves $L$. Then $N / E$ is NIP when equipped with its natural L-structure.

Proof. Let $c$ be a constant symbol and $f$ an $n$-ary function symbol. By $E$ preserving the language, we mean that for every $\left(a_{1}, \ldots, a_{n}\right)$ in $N^{n}$, whenever $a_{i E}=b_{i E}$ holds for every $i \in\{1, \ldots, n\}$ one has

$$
\left(f^{N}\left(a_{1}, \ldots, a_{n}\right)\right)_{E}=\left(f^{N}\left(b_{1}, \ldots, b_{n}\right)\right)_{E} .
$$

This way, the quotient space $N / E$ has a natural $L$-structure defined by putting

$$
c^{N / E}=c_{E}^{N} \quad \text { and } \quad f^{N / E}\left(a_{1 E}, \ldots, a_{n E}\right)=\left(f^{N}\left(a_{1}, \ldots, a_{n}\right)\right)_{E} .
$$

Since nice sets are externally definable, by a theorem of S. Shelah [She09], the structure $\left(M, L^{M}, N, E\right)$ in the language $L$ expanded by predicates for $N$ and $E$ is NIP. The domain and functions of the $L$-structure $N / E$ are interpretable in $\left(M, L^{M}, N, E\right)$, thus $N / E$ is also NIP.

As we shall only use this result in Theorem 4.4 for a quantifier-free formula $x y=y x$, we give a direct proof for that particular case: by induction on the complexity of an $L$-term $t\left(x_{1}, \ldots, x_{n}\right)$, for any $\left(a_{1}, \ldots, a_{n}\right) \in N^{n}$ one has

$$
t^{N / E}\left(a_{1 E}, \ldots, a_{n E}\right)=\left(t^{N}\left(a_{1}, \ldots, a_{n}\right)\right)_{E} .
$$

Let $E=\varphi(M, \overline{\mathbf{b}})$ and $N=\nu(M, \overline{\mathbf{c}})$ for some $L$-formulas $\varphi(x, y, \bar{z}), \nu(x, \bar{z})$ and tuples $\overline{\mathbf{b}}, \overline{\mathbf{c}}$ in M. For any quantifier-free $L \cup\{N, E\}$-formula $\phi(\bar{x})$, one defines the $L$-formula $\phi_{E}(\bar{x})$ by replacing any atomic subformula $t(\bar{x})=u(\bar{x})$ by $\varphi(t, u, \mathbf{b}) \wedge \nu\left(x_{1}, \overline{\mathbf{c}}\right) \wedge \cdots \wedge \nu\left(x_{n}, \overline{\mathbf{c}}\right)$. For any $\left(a_{1}, \ldots, a_{n}\right) \in N^{n}$, one has

$$
N / E \models \phi\left(a_{1 E}, \ldots, a_{n E}\right) \Longleftrightarrow \mathbf{M} \models \phi_{E}\left(a_{1}, \ldots, a_{n}\right) .
$$

It follows that, if the quantifier-free formula $\psi(\bar{x}, \bar{y})$ has the independence property in $M / E$, then the formula $\psi_{E}(\bar{x}, \bar{y})$ has the independence property in $\mathbf{M}$.

\section{Preliminaries on NIP Groups}

We now consider a NIP $L$-structure $M$ and a group $G$ definable in $M$.

\subsection{Descending chain conditions.}

Baldwin-Saxl chain condition 2.1 (see [BS76] or [Poi87]). Let $\left\{H_{i}: i \in I\right\}$ be a family of uniformly definable subgroups of $G$. There is $n \in \omega$ such that for all finite subsets $J \subset I$, there exists a finite subset $J_{n} \subset J$ of size at most $n$ such that

$$
\bigcap_{j \in J_{n}} H_{j}=\bigcap_{j \in J} H_{j} .
$$


We shall need the following stronger version. Given a subset $X \subset G$ and $n \in \omega$, we write $X^{0}=\{1\}$ and $X^{n}$ for the set of products $x_{1} x_{2} \cdots x_{n}$ of $n$ elements of $X$, and $X^{\times n}$ for the Cartesian product $X \times \cdots \times X$. We call $X$ symmetric if $X^{-1}=X$ and $1 \in X$. Given a pure set $Y$, when there is no ambiguity, we go on writing $Y^{n}$ for the Cartesian product $Y \times \cdots \times Y$.

Lemma 2.2 (a Baldwin Saxl chain condition for subsets). Let $\mathfrak{X}$ be a family of subsets of $G$. For every $X \in \mathfrak{X}$, let $X^{1 / 3} \subset G$ be definable with $\left(X^{1 / 3}\right)^{3} \subset X$. Let $\left\{X^{1 / 3}: X \in \mathfrak{X}\right\}$ be uniformly definable by a formula $\varphi(x, \bar{y})$ with $\operatorname{VC}\left(\varphi^{*}(x, \bar{y})\right)=n$. For every $X \in \mathfrak{X}$, let $X^{1 / 3 n} \subset G$ be symmetric (not necessarily definable) such that $\left(X^{1 / 3 n}\right)^{n} \subset X^{1 / 3}$. Then, for every $X_{1}, \ldots, X_{n+1} \in \mathfrak{X}$, there is $j \in\{1, \ldots, n+1\}$ with

$$
X_{1}^{1 / 3 n} \cap \cdots \cap X_{j-1}^{1 / 3 n} \cap X_{j+1}^{1 / 3 n} \cap \cdots \cap X_{n+1}^{1 / 3 n} \subset X_{1} \cap \cdots \cap X_{n+1} .
$$

Proof. Otherwise, there are $b_{1}, \ldots, b_{n+1}$ in $G$ and $X_{1}, \ldots, X_{n+1}$ in $\mathfrak{X}$ such that for all $j$ in $\{1, \ldots, n+1\}$, one has

$$
b_{j} \in\left(X_{1}^{1 / 3 n} \cap \cdots \cap X_{n+1}^{1 / 3 n}\right) \backslash X_{j} .
$$

Let $J \subset\{1, \ldots, n+1\}$ have elements $j_{1}<\cdots<j_{k}$ and let $b_{J}$ be the ordered product $b_{j_{1}} b_{j_{2}} \cdots b_{j_{k}}$. If $j \in\{1, \ldots, n+1\} \backslash J$, then $b_{J} \in\left(X_{j}^{1 / 3 n}\right)^{n}$ and so $b_{J} \in X_{j}^{1 / 3}$. On the other hand, if $J$ has elements

$$
j_{1}<\cdots<j_{i-1}<j<j_{i+1}<\cdots<j_{k},
$$

then $b_{J} \notin X_{j}^{1 / 3}$, for otherwise, we would have

$$
b_{j}=\left(b_{j_{i-1}}^{-1} \cdots b_{j_{1}}^{-1}\right) b_{J}\left(b_{j_{m}}^{-1} \cdots b_{j_{i+1}}^{-1}\right),
$$

and thus $b_{j} \in X_{j}$, a contradiction with (1). This shows that $V C\left(\varphi^{*}(x, \bar{y})\right) \geqslant n+1$, contradicting the hypothesis.

2.2. Nice subgroups. Let $H \subset G$ be a subgroup. $H$ is a type definable subgroup of $G$ if there is a partial type $\pi(x)$ with parameters in $G$ such that $H=\pi(G)$ and, for any elementary extension $\mathbf{G}$ of $G$, the set $\pi(\mathbf{G})$ is a subgroup of $\mathbf{G}$. We call $\pi$ a defining type for $H$.

Definition 2.3 (nice subgroup). We say that $H$ is a nice subgroup of $G$ if $H$ is a type definable subgroup of $G$ having a defining type $\pi(x)=\{\varphi(x, \bar{a}): \bar{a} \in A\}$ that is nice in $G$ and such that $\varphi(G, \bar{a})$ is symmetric for all $\bar{a}$ in $A$.

By Lemma 1.6, a nice subgroup is externally definable.

Example 2.4. A definable subgroup $H \subset G$ is nice. By the Baldwin Saxl chain condition, any intersection of uniformly definable subgroups of $G$ is nice. In particular, for any subset $A \subset G$ and subgroup $\mathfrak{A} \subset A u t(G)$, the subgroups $\bigcap_{a \in A} H^{a}, C_{G}(A)$ and $\bigcap_{\sigma \in \mathfrak{A}} H^{\sigma}$ are nice.

Counterexample 2.5 (A centraliser that is not nice). In an infinite extraspecial 3-group $K$, which is supersimple of rank 1 (see [MS08]) and whose conjugacy classes are all finite, choose $\left(a_{n}\right)_{n \geqslant 1}$ such that the chain of centralisers $C_{K}\left(a_{1}\right) \supset C_{K}\left(a_{1}, a_{2}\right) \supset C_{K}\left(a_{1}, a_{2}, a_{3}\right) \supset \cdots$ is strictly decreasing. The partial type $\bigcap_{n \geqslant 1} C_{K}\left(a_{n}\right)$ is not nice as $\left[K: C_{K}\left(a_{n}\right)\right] \leqslant 3$ for every $n$. Nor is it equivalent to a nice partial type, for otherwise, by the Compactness 
theorem, one could find a definable infinite subset $X \subset K$ with infinitely many pairwise disjoint left translates, contradicting the fact that $K$ has rank 1.

Example 2.6. In an $\omega$-saturated elementary extension $\mathfrak{R}$ of the field $\mathbf{R}$, the subgroup of infinitesimal numbers is nicely defined in the language $(+, \leqslant)$. In the language of fields, the intersection of the Euclidian balls $\left\{x \in \mathfrak{R}^{n}:\|x\|_{2}<1 / k\right\}$ is a nice subgroup of $\mathfrak{R}^{n}$. It is also the intersection of the family $\mathfrak{H}$ of half hyperplanes of equations $a_{1} x_{1}+\cdots+a_{n} x_{n} \leqslant a_{n+1}$ where $a_{1}, \ldots, a_{n+1}$ range over $\mathbf{Q}$ with $a_{n+1}>0$. In $\operatorname{GL}_{n}(\mathfrak{R})$, considered as a group interpretable in the ring $\mathrm{M}_{\mathrm{n}}(\mathfrak{R})$, the subgroup of elements that are infinitesimally close to 1 is nice, being the intersection of the neighbourhoods $\left\{1+x:\|x\|_{2}<1 / k\right\}$.

Example 2.7. Let $\mathfrak{Z}_{p}$ be an $\omega$-saturated elementary extension of the ring $\mathbf{Z}_{p}$ of $p$-adics integers. The infinitesimal numbers form a nice subgroup of $\mathfrak{Z}_{p}$, defined by the intersection of the subgroups $p^{k} \mathfrak{Z}_{p}$. In $\mathrm{GL}_{\mathrm{n}}\left(\mathfrak{Z}_{p}\right)$, as a group interpretable in the ring $\mathrm{M}_{\mathrm{n}}\left(\mathfrak{Z}_{p}\right)$, the intersection of the congruence subgroups $1+p^{k} \mathrm{M}_{\mathrm{n}}\left(\mathfrak{Z}_{p}\right)$ is a nice subgroup.

2.3. Normaliser. For any two subgroups $H, K \subset G$, we write

$$
H^{K}=\bigcap_{g \in K} H^{g}
$$

for the $K$-core of $H$. When $G$ is stable, if $H$ is definable, then $H^{K}$ is definable, and hence so is $N_{G}\left(H^{K}\right)$. When $G$ is NIP, the situation is far less straightforward. $H^{K}$ is merely $K$-type definable and its normaliser has no obvious reason to be even type definable.

Lemma 2.8. Let $H \subset G$ be a nice subgroup with defining type $\{\varphi(x, \bar{b}): \bar{b} \in B\}$ and let $n=V C\left(\varphi^{*}(x, \bar{y})\right)$. For any subset $A \subset G$, the subgroup $H^{A} \subset G$ is nice, with defining type $\left\{\bigwedge_{1 \leqslant i \leqslant n} \varphi\left(x^{a_{i}}, \bar{b}\right): \bar{b} \in B, a_{1}, \ldots, a_{n} \in A\right\}$.

Proof. Let $\mathfrak{F}$ be the family of uniformly definable sets $\{\varphi(G, \bar{b}): \bar{b} \in B\}$. As $\pi(\mathbf{G})^{3} \subset \pi(\mathbf{G})$ for every elementary extension $\mathbf{G}$ of $G$, by the Compactness Theorem, for every element $X$ of $\mathfrak{F}$, there are finitely many $X_{1}, \ldots, X_{m}$ in $\mathfrak{F}$ such that $\left(X_{1} \cap \cdots \cap X_{m}\right)^{3} \subset X$. As $\mathfrak{F}$ is a filter, there is an element of $\mathfrak{F}$, which we write $X^{1 / 3}$, such that $X^{1 / 3} \subset X_{1} \cap \cdots \cap X_{m}$. Similarly, for every non-zero $n \in \omega$, there is $X^{1 / 3 n} \in \mathfrak{F}$ such that

$$
\left(X^{1 / 3 n}\right)^{n} \subset X^{1 / 3} \subset X
$$

Let $\psi\left(x ; \bar{y}, y_{k+1}\right)$ be the formula $\varphi\left(x^{y_{k+1}}, \bar{y}\right)$ and $n=V C\left(\psi^{*}\left(x ; \bar{y}, y_{k+1}\right)\right)$ where $\bar{y}=\left(y_{1}, \ldots, y_{k}\right)$. By Lemma 2.2 applied to the family $\mathfrak{G}=\left\{X^{a}: a \in A, X \in \mathfrak{F}\right\}$, for every $X_{1}, \ldots, X_{n+1}$ in $\mathfrak{G}$, there are $Y_{1}, \ldots, Y_{n}$ in $\mathfrak{G}$ such that

$$
Y_{1} \cap \cdots \cap Y_{n} \subset X_{1} \cap \cdots \cap X_{n+1}
$$

It follows that the family $\left\{X_{1} \cap \cdots \cap X_{n}: X_{i} \in \mathfrak{G}\right\}$ is a filter. 
We adopt the following conventions for writing down formulas. For every non-zero $k \in \omega$ and formulas $\varphi(x, \bar{u}), \phi(x, \bar{v})$, we write

$$
\begin{array}{rll}
\varphi(x, \bar{u})^{k} & \text { for } & \exists x_{1} \ldots \exists x_{k}\left(\varphi\left(x_{1}, \bar{u}\right) \wedge \cdots \wedge \varphi\left(x_{k}, \bar{u}\right) \wedge x=x_{1} x_{2} \cdots x_{k}\right), \\
\varphi(x, \bar{v})^{z} & \text { for } & \exists y\left(\varphi(y, \bar{v}) \wedge x=y^{z}\right), \\
\varphi(x, \bar{u}) \subset_{x} \phi(x, \bar{v}) & \text { for } & \forall x(\varphi(x, \bar{u}) \rightarrow \phi(x, \bar{v})) .
\end{array}
$$

Lemma 2.9. Let $G$ be $\kappa$-saturated with $\kappa>\aleph_{0}$. Let $A, N \subset G$ two subgroups with $|A|<\kappa$ and $|N|<\kappa$. Let $H \subset G$ be a nice subgroup with a countable defining type $\{\varphi(x, \bar{b}): \bar{b} \in B\}$. Let $n=\operatorname{VC}\left(\varphi^{*}(x, \bar{y})\right)$. Assume that $A \subset H$ and $A^{N} \subset A$. For any $\bar{b} \in B$ and $a_{1}, \ldots, a_{n}$ in $G$ such that $A \subset \bigcap_{1 \leqslant i \leqslant n} \varphi(G, \bar{b})^{a_{i}}$, there are a nice subgroup $K \subset G$ with countable defining type $\{\varphi(x, \bar{c}): \bar{c} \in C\}$, some $\bar{c} \in C$ and $\alpha_{1}, \ldots, \alpha_{n}$ in $G$ such that, for every $g \in N$,

$$
\left(\bigcap_{i=1}^{n} \varphi(G, \bar{c})^{\alpha_{i}}\right)^{g} \subset \bigcap_{i=1}^{n} \varphi(G, \bar{b})^{a_{i}} \quad \text { and } \quad A \subset K \cap K^{\alpha_{1}} \cap \cdots \cap K^{\alpha_{n}} .
$$

Proof. Let $\left(\bar{b}_{i}\right)_{i \in \omega}$ be an enumeration of $B$ such that $\varphi\left(G, \bar{b}_{i+1}\right)^{2} \subset \varphi\left(G, \bar{b}_{i}\right)$ for all $i \in \omega$. We consider the partial type over $A$

$$
\rho\left(\left(\bar{y}_{i}\right)_{i \in \omega}\right)=\left\{\varphi\left(x, \bar{y}_{i+1}\right)^{2} \subset_{x} \varphi\left(x, \bar{y}_{i}\right), \varphi\left(a, \bar{y}_{i}\right): i \in \omega, a \in A\right\} .
$$

Note that for all $m \in \omega$, the sequence $\left(\bar{b}_{m+i}\right)_{i \in \omega}$ satisfies $\rho$. We consider the partial type $\pi\left(x_{1}, \ldots, x_{n}, \bar{y}_{0}\right)$ with parameters in $A \cup N \cup\left\{a_{1}, \ldots, a_{n}, \bar{b}\right\}$, defined by

$$
\left\{\bigwedge_{i=1}^{n} \varphi\left(x, \bar{y}_{0}\right)^{x_{i}} \subset_{x}\left(\bigwedge_{i=1}^{n} \varphi(x, \bar{b})^{a_{i}}\right)^{g}, \bigwedge_{i=1}^{n} \varphi\left(a, \bar{y}_{0}\right)^{x_{i}} \wedge \varphi\left(a, \bar{y}_{0}\right): g \in N, a \in A\right\} .
$$

By Lemma 2.8, the type $\pi\left(x_{1}, \ldots, x_{n}, \bar{y}_{0}\right)$ is finitely satisfiable in $(N)^{\times n} \times B$. It follows that $\pi\left(x_{1}, \ldots, x_{n}, \bar{y}_{0}\right) \cup \rho\left(\left(\bar{y}_{i}\right)_{i \in \omega}\right)$ is satisfiable. As $G$ is $\kappa$-saturated, $\pi \cup \rho$ has a realisation $\left(\alpha_{1}, \ldots, \alpha_{n},\left(\bar{c}_{i}\right)_{i \in \omega}\right)$ in $G$. We put $C=\left\{\bar{c}_{i}: i \in \omega\right\}$.

Theorem 2.10 (normalising a nice envelope). Let $G$ be $\kappa$-saturated with $\kappa>\aleph_{0}$ and $H \subset G$ a nice subgroup defined by a partial type of size $<\kappa$. Let $A \subset H$ and $N_{A} \subset N_{G}(A)$ any subgroups with $|A|<\kappa$ and $\left|N_{A}\right|<\kappa$. There are nice subgroups $K, N_{K} \subset G$ defined by countable partial types such that

$$
A \subset K \subset H \quad \text { and } \quad N_{A} \subset N_{K} \subset N_{G}(K) .
$$

Proof. Let $\{\varphi(x, \bar{b}): \bar{b} \in B\}$ be a defining type for $H$. Note that $H$ is the intersection of nice subgroups $H_{i} \subset G$ having a countable defining type. We fix some $\bar{\beta} \in B$. There is some index $i$ and some $\bar{b} \in B$ such that

$$
H_{i} \subset \varphi(G, \bar{b}) \subset \varphi(G, \bar{b})^{2} \subset \varphi(G, \bar{\beta}) .
$$

We apply the previous lemma with $a_{1}=\cdots=a_{n}=1$ and put

$$
X_{0}=\varphi(G, \bar{\beta}) \quad \text { and } \quad X_{1}=\bigcap_{i=1}^{n} \varphi(G, \bar{c})^{\alpha_{i}} .
$$


One has $X_{1}^{g} \subset X_{0}$ for any $g \in N_{A}$ and $X_{1} X_{1} \subset X_{0}$. Let $\bar{\gamma}$ be an element of $C$ such that

$$
\left(\bigcap_{i=1}^{n} \varphi(G, \bar{\gamma})^{\alpha_{i}}\right)^{2} \subset X_{1}
$$

and put $Y_{1}=\bigcap_{i=1}^{n} \varphi(G, \bar{\gamma})^{\alpha_{i}}$. As $A \subset Y_{1}$, and as the countable type $\{\varphi(x, \bar{c}): \bar{c} \in C\}$ provided by Lemma 2.9 defines a nice subgroup $H_{1} \subset G$ with $A \subset H_{1}$, one can apply Lemma 2.9 again to $H_{1}$ and $Y_{1}$. By induction, one finds an infinite decreasing chain of definable subsets $X_{0} \supset X_{1} \supset X_{2} \supset \cdots$ of $G$ such that for every $i \in \omega$ and $g \in N_{A}$, one has

$$
X_{i+1}^{g} \subset X_{i}, \quad A \subset X_{i} \text { and } X_{i+1}^{2} \subset X_{i} .
$$

As $n$ depends on $\varphi(x, y)$ only, the family $\left\{X_{i}: i \in \omega\right\}$ is uniformly defined by the formula

$$
\psi\left(x, y_{1}, \ldots, y_{n+1}\right)=\bigwedge_{1 \leqslant i \leqslant n} \varphi\left(x^{y_{i}}, y_{n+1}\right),
$$

where $y_{1}, \ldots, y_{n+1}$ are replaced by parameters. By compactness, there is a family $\left(Y_{i}\right)_{i \in \mathbf{Q}}$ of uniformly definable subsets of $G$ defined by $\psi\left(x, y_{1}, \ldots, y_{n+1}\right)$, such that for all rational numbers $p<q$, all $g \in N_{A}$ and all $\bar{\beta} \in B$, one has

$$
Y_{p}^{g} \subset Y_{q}, \quad A \subset Y_{p} \subset \varphi(G, \bar{\beta}), \quad \text { and } \quad Y_{p} Y_{p} \subset Y_{q} .
$$

We put $Y_{p}=\psi\left(G, \bar{b}_{p}\right)$ for some tuple $\bar{b}_{p}$. By compactness and Ramsey's Theorem, we may assume that the sequence $\left(\bar{b}_{p}\right)_{p \in \mathbf{Q}}$ is indiscernible over the empty set. We define

$$
K=\bigcap_{p \in \mathbf{Q}} Y_{p} \quad \text { and } \quad N_{K}=\bigcap_{\substack{(p, q) \in \mathbf{Q}^{2} \\ p<q}}\left\{x \in G: Y_{p}^{x} \subset Y_{q} \text { and } Y_{p}^{x^{-1}} \subset Y_{q}\right\} .
$$

It is straightforward that $K$ is a nice subgroup, that $K \subset H$, that $N_{A} \subset N_{K} \subset N_{G}(K)$ and that $N_{K}$ is symmetric. For any rational numbers $p<r<q$, one has

$$
\left(\left\{x \in G: Y_{p}^{x} \subset Y_{r}\right\} \cap\left\{x \in G: Y_{r}^{x} \subset Y_{q}\right\}\right)^{2} \subset\left\{x \in G: Y_{p}^{x} \subset Y_{q}\right\} .
$$

It follows that $N_{K}$ is a subgroup of $G$. To finish the proof of Theorem 2.10, we only need to show that $N_{K}$ is a nice subgroup. For any $p<q$, we define $\langle p, q\rangle$ putting

$$
\langle p, q\rangle=\left\{x \in G: Y_{p}^{x} \subset Y_{q} \text { and } Y_{p}^{x^{-1}} \subset Y_{q}\right\},
$$

and for any $r_{0}<r_{1}<\cdots<r_{m}$, we define $\left\langle r_{0}, \ldots, r_{m}\right\rangle$ by

$$
\left\langle r_{0}, \ldots, r_{m}\right\rangle=\left\langle r_{0}, r_{1}\right\rangle \cap\left\langle r_{1}, r_{2}\right\rangle \cdots \cap\left\langle r_{m-1}, r_{m}\right\rangle .
$$

Note that $\left\langle r_{0}, \ldots, r_{m}\right\rangle^{m} \subset\left\langle r_{0}, r_{m}\right\rangle$. Let $\phi(x, \bar{y})$ be the formula defining uniformly the sets $\langle p, q\rangle$, and let $m=V C\left(\phi^{*}\left(x, \bar{y}_{1}\right) \wedge \phi^{*}\left(x, \bar{y}_{2}\right) \wedge \phi^{*}\left(x, \bar{y}_{3}\right)\right)$. Let $r_{0}<r_{1}<\cdots<r_{2 m+1}$ be an ordered sequence of $2 m+2$ rational numbers. By Lemma 2.2, there is $i \leqslant 2 m$ such that

$$
\begin{aligned}
\left\langle r_{0}, r_{1}\right\rangle^{1 / 3 m} \cap \cdots \cap\left\langle r_{i-2}, r_{i-1}\right\rangle^{1 / 3 m} \cap\left\langle r_{i+2}, r_{i+3}\right\rangle^{1 / 3 m} \cap \cdots \cap\left\langle r_{2 m}, r_{2 m+1}\right\rangle^{1 / 3 m} & \\
& \subset\left\langle r_{0}, r_{1}\right\rangle \cap\left\langle r_{2}, r_{3}\right\rangle \cap \cdots \cap\left\langle r_{2 m}, r_{2 m+1}\right\rangle .
\end{aligned}
$$

To simplify notations, let us assume that $i=2$. The above equation yields in particular

$$
\left\langle r_{0}, r_{1}\right\rangle^{1 / 3 m} \cap\left\langle r_{4}, r_{5}\right\rangle^{1 / 3 m} \cap \cdots \cap\left\langle r_{2 m}, r_{2 m+1}\right\rangle^{1 / 3 m} \subset\left\langle r_{2}, r_{3}\right\rangle
$$


As the sequence $\left(\bar{b}_{p}\right)_{p \in \mathbf{Q}}$ is indiscernible, for any rational numbers $p_{0}<p_{1}<\cdots<p_{2 m+1}$, and any $p_{i}=p_{0}^{i, i+1}<p_{1}^{i, i+1}<\cdots<p_{3 m-1}^{i, i+1}<p_{3 m}^{i, i+1}=p_{i+1}$, one has

$$
\left\langle p_{0}^{0,1}, \ldots, p_{3 m}^{0,1}\right\rangle \cap\left\langle p_{0}^{4,5}, \ldots, p_{3 m}^{4,5}\right\rangle \cap \cdots \cap\left\langle p_{0}^{2 m, 2 m+1}, \ldots, p_{3 m}^{2 m, 2 m+1}\right\rangle \subset\left\langle p_{2}, p_{3}\right\rangle .
$$

In particular, by density of $\mathbf{Q}$, any finite intersection of subsets of the form $\langle p, q\rangle$ for $p<q$ contains an intersection of $3 \mathrm{~m}^{2}$ sets of the same form, so that if $\mathfrak{F}$ denotes the family $\{\langle p, q\rangle$ : $p<q\}$, then the family $\left\{\bigcap \mathfrak{F}_{m}: \mathfrak{F}_{m} \subset \mathfrak{F}\right.$ and $\left.\left|\mathfrak{F}_{m}\right| \leqslant 3 m^{2}\right\}$ is a uniform filter defining $N_{K}$ as well.

Remark 2.11. If $H$ is the intersection of uniformly definable groups, then $K$ is the intersection of uniformly definable subgroups, but we do not see any obvious reason why $N_{K}$ would be the intersection of uniformly definable subgroups.

With a similar proof, we get:

Theorem 2.12. Let $G$ be $\kappa$-saturated with $\kappa>\aleph_{0}$ and $H \subset G$ a nice subgroup defined by a partial type of size $<\kappa$. Let $A \subset H$ and $N_{A} \subset N_{G}(A)$ two subgroups that are the reunion of two families of cardinality $<\kappa$ of uniformly definable subsets of $G$. There are nice subgroups $K, N_{K} \subset G$ defined by countable types such that

$$
A \subset K \subset H \quad \text { and } \quad N_{A} \subset N_{K} \subset N_{G}(K) .
$$

\section{External AND Discernible SUbGroups}

Let $G$ be an infinite group, $H=\varphi(G, \overline{\mathbf{c}})$ an externally definable subgroup of $G$ and $\mathbf{G}$ a fixed $|G|^{+}$-saturated elementary extension of $G$. Every $\overline{\mathbf{b}}$ realising the $\varphi^{*}$-type of $\overline{\mathbf{c}}$ over $G$ satisfies $\varphi(G, \overline{\mathbf{c}})=\varphi(G, \overline{\mathbf{b}})$, so $H$ is externally definable with parameters in G. The group $H$ need not be the trace on $G$ of a definable subgroup of $\mathbf{G}$ though: consider the example of a convex proper additive subgroup of an elementary extension of $\mathbf{R}$.

Definition 3.1 (external subgroup). A subgroup $H \subset G$ is external if there is a definable subgroup $\mathbf{H} \subset \mathbf{G}$ such that $H=\mathbf{H} \cap G$. If the elements of $\mathbf{H}$ satisfy a set $\mathrm{P}$ of quantifier-free formulas with parameters in $G$, we say that $H$ is external as a P-group.

Definition 3.2 (discernible subgroup). A subgroup $H \subset G$ is discernible if there is an elementary extension $\mathbf{G}$ of $G$ and a nice subgroup $\mathbf{H} \subset \mathbf{G}$ such that $H=\mathbf{H} \cap G$. If the elements of $\mathbf{H}$ satisfy a set $\mathrm{P}$ of quantifier-free formulas with parameters in $G$, we say that $H$ is discernible as a P-group.

Lemma 3.3. A discernible subgroup of $G$ is externally definable.

Proof. Let $H=\mathbf{H} \cap G$ be discernible. By Lemma 1.6, $\mathbf{H}$ is externally definable, so $H$ also.

Lemma 3.4. A discernible subgroup $H=\mathbf{H} \cap G$ is the trace over $G$ of a nice subgroup $\mathbf{K} \subset \mathbf{H}$ defined by a countable partial type. 
Proof. Let $\pi(x)=\{\varphi(x, \bar{b}): \bar{b} \in \mathbf{B}\}$ be a defining type for $\mathbf{H}$. Let $\mathbf{G}_{1}$ be a $|\mathbf{G}|^{+}$-saturated elementary extension of $\mathbf{G}$. Let $\bar{b}$ in $\mathbf{B}$. As $\pi\left(\mathbf{G}_{1}\right)^{2} \subset \varphi\left(\mathbf{G}_{1}, \bar{b}\right)$, by the Compactness Theorem, there are $\bar{b}_{1}, \ldots, \bar{b}_{n}$ in $\mathbf{B}$ such that

$$
\left(\varphi\left(\mathbf{G}_{1}, \bar{b}_{1}\right) \cap \cdots \cap \varphi\left(\mathbf{G}_{1}, \bar{b}_{n}\right)\right)^{2} \subset \varphi\left(\mathbf{G}_{1}, \bar{b}\right) .
$$

As $\pi\left(\mathbf{G}_{1}\right)$ is nice, there is $\bar{c}$ in $\mathbf{B}$ such that

$$
\varphi\left(\mathbf{G}_{1}, \bar{c}\right) \subset \varphi\left(\mathbf{G}_{1}, \bar{b}_{1}\right) \cap \cdots \cap \varphi\left(\mathbf{G}_{1}, \bar{b}_{n}\right) .
$$

Thus, the following partial type $\rho\left(\bar{y}_{0}, \bar{y}_{1}, \ldots, \bar{y}_{n}, \ldots\right)$ over $H \cup \mathbf{B}$

$$
\left\{\varphi\left(x, \bar{y}_{i+1}\right)^{2} \subset_{x} \varphi\left(x, \bar{y}_{i}\right), \varphi\left(x, \bar{y}_{0}\right) \subset_{x} \varphi(x, \bar{b}), \varphi\left(h, \bar{y}_{i}\right): i \in \omega, h \in H, \bar{b} \in \mathbf{B}\right\}
$$

is finitely satisfiable in $\mathbf{B}$, hence realised by some sequence $\left(\overline{\mathbf{a}}_{0}, \overline{\mathbf{a}}_{0}, \ldots\right)$ of elements of $\mathbf{G}_{1} \succ \mathbf{G}$. Putting $\pi(x)=\left\{\varphi\left(x, \overline{\mathbf{a}}_{i}\right): i \in \omega\right\}$, one has $H=\pi\left(\mathbf{G}_{1}\right) \cap G$.

If $H$ is discernible as a P-subgroup of $G$ and defined by the countable partial type $\pi(x)=$ $\left\{\varphi\left(x, \mathbf{a}_{i}\right): i \in \omega\right\}$, then replacing $\left(\mathbf{a}_{i}\right)_{i \in \omega}$ by parameters $\left(\mathbf{b}_{i}\right)_{i \in \omega}$ sharing the same type over $G$ changes neither $H$ nor the first order consequences (with parameters in $G$ ) of $\pi(x)$. In particular, $H$ is the trace on $G$ of a nice subgroup $\mathbf{H} \subset \mathbf{G}$ whose elements satisfy P.

An external subgroup of $G$ is a discernible subgroup of $G$. The subgroup of infinitesimal numbers of an elementary extension $\mathfrak{R}$ of $\mathbf{R}$ is a discernible subgroup, an externally definable subset, but not an external subgroup of $\mathfrak{R}$.

Lemma 3.5. Let $H \subset G$ be the intersection of a family of uniformly external subgroups of $G$ with defining formula $\varphi(x, \bar{y})$. Then $H$ is an external subgroup of $G$ with defining formula $\varphi\left(x, \bar{y}_{1}\right) \wedge \cdots \wedge \varphi\left(x, \bar{y}_{n}\right)$.

Proof. By the Baldwin Saxl condition, $H$ is a discernible subgroup of $G$. We can thus apply the proof of Lemma 3.3, adding to the partial type $\pi(\bar{y})$ a formula $\psi(\bar{y})$ saying that $\varphi(x, \bar{y})$ defines a subgroup of $G$.

Lemma 3.6. If $G$ is stable, a discernible subgroup $H \subset G$ is definable. If $H$ is the trace over $G$ of a group $\mathbf{H}$ defined by the nice type $\{\varphi(x, \overline{\mathbf{a}}): \overline{\mathbf{a}} \in \mathbf{A}\}$, there are $\bar{a}_{1}, \ldots, \bar{a}_{n}$ in $G$ such that $\varphi\left(G, \bar{a}_{1}\right) \cap \cdots \cap \varphi\left(G, \bar{a}_{n}\right)$ is a subgroup of $H$ of finite index.

Proof. As $\mathbf{G}$ does not have the order property, there is $\mathbf{a}$ in $\mathbf{A}$ such that $\mathbf{H}=\varphi(\mathbf{G}, \mathbf{a})$. Let $\psi(y)$ be a formula stating that $\varphi(G, y)$ is a subgroup of $G$. The $\varphi^{*} \wedge \psi$-type of a over $G$ is definable by a positive Boolean combination of formulas of the form $\varphi(x, g) \wedge \psi(g)$ for $g$ in $G$ by [HH84, Corollary 2.8], hence covered by a finite union of subgroups of $G$. By Neumann's Lemma [Neu54], one of these subgroups must have finite index in $H$.

Theorem 3.7 (finding external subgroups). Let $G$ a NIP group, G a $|G|^{+}$-saturated elementary extension of $G$ and $H=\mathbf{H} \cap G$ an external subgroup of $G$.

(1) There is $n \in \omega$ such that for every $A \subset G$, there are $\mathbf{a}_{1}, \ldots, \mathbf{a}_{n}$ in $\mathbf{G}$ such that

$$
C_{G}(A)=C_{G}\left(\mathbf{a}_{1}, \ldots, \mathbf{a}_{n}\right) .
$$


(2) There is an abelian definable subgroup $\mathbf{Z} \subset \mathbf{G}$ such that,

$$
Z(H)=\mathbf{Z} \cap G .
$$

(3) For every $n \in \omega$, there is a definable subgroup $\mathbf{K}$ of $\mathbf{G}$ such that

$$
H=\mathbf{K} \cap G \quad \text { and } \quad Z_{n}(H)=Z_{n}(\mathbf{K}) \cap G .
$$

(4) There is $n \in \omega$ such that for every $A \subset G$, there are $\mathbf{a}_{1}, \ldots, \mathbf{a}_{n}$ in $\mathbf{G}$ such that

$$
H^{A}=\mathbf{H}^{\mathbf{a}_{1}} \cap \cdots \cap \mathbf{H}^{\mathbf{a}_{n}} \cap G .
$$

Proof. (1) By Baldwin Saxl's chain condition, $C_{G}(A)$ is defined by a nice partial type consisting of uniformly definable subgroups. It is thus an external subgroup by Lemma 3.5.

(2) By the Baldwin Saxl condition, there is $n \in \omega$ such that the centraliser of any finite subset of $\mathbf{G}$ is the centraliser of $n$ elements. By the Compactness theorem and the saturation assumption, there is an $n$-tuple $\overline{\mathbf{h}}$ in $\mathbf{G}$ such that $Z(H) \subset C_{\mathbf{H}}(\overline{\mathbf{h}}) \subset C_{\mathbf{H}}(H)$. It follows that $Z\left(C_{\mathbf{H}}(\overline{\mathbf{h}})\right)$ contains $Z(H)$, hence $Z(H)=G \cap Z\left(C_{\mathbf{H}}(\overline{\mathbf{h}})\right)$.

(3) The following Claim is inspired by [dA13, Lemma 2.1]:

Claim 1. Let $A, B \subset G$ be two subgroups and $\mathbf{D} \subset \mathbf{G}$ a definable subgroup normalised by both $A$ and $B$ such that $[A, B] \subset \mathbf{D}$. There are two definable subgroups $\mathbf{A}, \mathbf{B} \subset \mathbf{G}$ containing $A$ and $B$ respectively such that $[\mathbf{A}, \mathbf{B}] \subset \mathbf{D}$.

Proof of Claim 1. For any subset $C \subset \mathbf{G}$, we define the subgroups $\mathbf{A}(C), \mathbf{B}(C) \subset \mathbf{G}$ by

$$
\begin{aligned}
& \mathbf{A}(C)=\bigcap_{\substack{c \in C \\
[A, c] \subset \mathbf{D}}}\left\{x \in N_{\mathbf{G}}(\mathbf{D}):[x, c] \subset \mathbf{D}\right\}, \\
& \mathbf{B}(C)=\bigcap_{\substack{c \in C \\
[c, B] \subset \mathbf{D}}}\left\{y \in N_{\mathbf{G}}(\mathbf{D}):[c, y] \subset \mathbf{D}\right\},
\end{aligned}
$$

and claim that there is a finite $C \subset \mathbf{G}$ such that $[\mathbf{A}(A \cup B \cup C), \mathbf{B}(A \cup B \cup C)] \subset \mathbf{D}$. Otherwise, by induction, one could build two sequences $\left(a_{n}\right)_{n \in \omega}$ and $\left(b_{n}\right)_{n \in \omega}$ such that for every $n \in \omega, a_{n} \in \mathbf{A}\left(A \cup B \cup\left\{a_{k}, b_{k}: k<n\right\}\right)$ and $b_{n} \in \mathbf{B}\left(A \cup B \cup\left\{a_{k}, b_{k}: k<n\right\}\right)$ but $\left[a_{n}, b_{n}\right] \notin \mathbf{D}$. It would follow that $\left[a_{i}, b_{j}\right] \in \mathbf{D}$ if and only if $i \neq j$, so that the sequence $\left(C_{\mathbf{G}}\left(a_{n} \mathbf{D}\right)\right)_{n \in \omega}$ would not satisfy the Baldwin Saxl chain condition since for every $j \leqslant n$,

$$
b_{j} \in\left(\bigcap_{\substack{1 \leqslant i \leqslant n \\ i \neq j}} C_{\mathbf{G}}\left(a_{i} \mathbf{D}\right)\right) \backslash\left(\bigcap_{1 \leqslant i \leqslant n} C_{\mathbf{G}}\left(a_{i} \mathbf{D}\right)\right) .
$$

By the Compactness Theorem, there is a finite tuple $\bar{c}$ such that $[\mathbf{A}(\bar{c}), \mathbf{B}(\bar{c})] \subset \mathbf{D}$. We consider $\mathbf{A}=\mathbf{A}(\bar{c})$ and $\mathbf{B}=\mathbf{B}(\bar{c})$.

We prove (3) by induction on $n$. For $n=0$, there is nothing to show. If there is a definable subgroup $\mathbf{H} \subset \mathbf{G}$ such that $H=\mathbf{H} \cap G$ and $Z_{n}(H)=Z_{n}(\mathbf{H}) \cap G$, as $\left[Z_{n+1}(H), H\right] \subset$ $Z_{n}(\mathbf{H})$, by Claim 1, there are two definable subgroups $\mathbf{Z}_{n+1}, \mathbf{H}_{n+1} \subset \mathbf{G}$ containing $Z_{n+1}(H)$ and $H$ respectively such that $\left[\mathbf{Z}_{n+1}, \mathbf{H}_{n+1}\right] \subset Z_{n}(\mathbf{H})$. Replacing $\mathbf{H}_{n+1}$ by $\mathbf{H}_{n+1} \cap \mathbf{H}$ and $\mathbf{Z}_{n+1}$ by $\mathbf{Z}_{n+1} \cap \mathbf{H}_{n+1}$, we may assume that $\mathbf{H}_{n+1} \subset \mathbf{H}$ and $\mathbf{Z}_{n+1} \subset \mathbf{H}_{n+1}$. It follows that $\left[\mathbf{Z}_{n+1}, \mathbf{H}_{n+1}\right] \subset Z_{n}\left(\mathbf{H}_{n+1}\right)$, so that $Z_{n+1}\left(\mathbf{H}_{n+1}\right)$ contains $\mathbf{Z}_{n+1}$, hence $Z_{n+1}(H)$. One thus has

$$
H=\mathbf{H}_{n+1} \cap G \quad \text { and } \quad Z_{n+1}(H)=Z_{n+1}\left(\mathbf{H}_{n+1}\right) \cap G \text {. }
$$


(4) Follows from Baldwin Saxl's chain condition and Lemma 3.5.

Theorem 3.8 (finding discernible subgroups). Let $G$ be a NIP group, $\mathbf{G}$ a $|G|^{+}$-saturated, $|G|^{+}$-homogeneous elementary extension of $G$ and $H=\mathbf{H} \cap G$ a discernible subgroup of $G$.

(1) There are $n \in \omega$ and a nice subgroup $\mathbf{K} \subset \mathbf{G}$ such that for every $A \subset G$, there are $\mathbf{a}_{1}, \ldots, \mathbf{a}_{n}$ in $\mathbf{G}$ with

$$
H=\mathbf{K} \cap G \quad \text { and } \quad H^{A}=\mathbf{K}^{\mathbf{a}_{1}} \cap \cdots \cap \mathbf{K}^{\mathbf{a}_{n}} \cap G .
$$

(2) There are $n \in \omega$ and a nice subgroup $\mathbf{K} \subset \mathbf{G}$ such that for every $A \subset G$ and $\mathfrak{A} \subset \operatorname{Aut}(G / A)$, there are $\boldsymbol{\sigma}_{1}, \ldots, \boldsymbol{\sigma}_{n}$ in $\operatorname{Aut}(\mathbf{G} / A)$ with

$$
H=\mathbf{K} \cap G \quad \text { and } \quad \bigcap_{\sigma \in \mathfrak{A}} H^{\sigma}=\mathbf{K}^{\sigma_{1}} \cap \cdots \cap \mathbf{K}^{\sigma_{n}} \cap G .
$$

(3) There are nice subgroups $\mathbf{K} \subset \mathbf{H}$ and $\mathbf{N} \subset N_{\mathbf{G}}(\mathbf{K})$ of $\mathbf{G}$ such that

$$
H=\mathbf{K} \cap G \quad \text { and } \quad N_{G}(H)=\mathbf{N} \cap G .
$$

Proof. (1) Let $\{\varphi(x, \bar{b}): \bar{b} \in \mathbf{B}\}$ be a defining type for $\mathbf{H}$ and $n=V C\left(\varphi^{*}(x, \bar{y})\right)$. By Lemma 2.8 , the type $\pi_{A}\left(y_{1}, \ldots, y_{n},\left(x_{i}\right)_{i \in \omega}\right)$ defined by

$$
\begin{aligned}
\left\{\varphi\left(x, \bar{x}_{i+1}\right)^{2} \subset_{x} \varphi\left(x, \bar{x}_{i}\right), \varphi\left(x, \bar{x}_{i}\right) \subset_{x} \varphi(x, \bar{b}), \varphi\left(h, \bar{x}_{i}\right), \bigwedge_{1 \leqslant j \leqslant n} \varphi\left(k, \bar{x}_{i}\right)^{y_{j}},\right. \\
\left.\left(\bigwedge_{1 \leqslant j \leqslant n} \varphi\left(x, \bar{x}_{i}\right)^{y_{j}}\right) \subset_{x} \varphi(x, \bar{b})^{a}: h \in H, k \in H^{A}, a \in A, \bar{b} \in \mathbf{B}, i \in \omega\right\}
\end{aligned}
$$

is finitely satisfiable in $A^{\times n} \times \mathbf{B}^{\omega}$. Let $\left(\mathbf{a}_{1}, \ldots, \mathbf{a}_{n},\left(\mathbf{c}_{i}\right)_{i \in \omega}\right)$ be a realisation of $\pi_{A}$ in $\mathbf{G}$. The type $\nu(x)=\left\{\varphi\left(x, \overline{\mathbf{c}}_{i}\right): i \in \omega\right\}$ is nice, and one has

$$
H=\nu(\mathbf{G}) \cap G \quad \text { and } \quad H^{A}=\nu(\mathbf{G})^{\mathbf{a}_{1}} \cap \cdots \cap \nu(\mathbf{G})^{\mathbf{a}_{n}} \cap G .
$$

By another compactness argument, considering the union of the types $\pi_{A}\left(y_{1, A}, \ldots, y_{n, A},\left(x_{i}\right)_{i \in \omega}\right)$ when $A$ ranges among all subsets of $G$, one can find a group $\nu(\mathbf{G})$ that does not depend on the set $A$.

(2) By Lemma 3.4, we may change $\mathbf{H}$ and assume that it is defined by a countable type $\left\{\varphi\left(x, \overline{\mathbf{b}}_{i}\right): i \in \omega\right\}$. Without loss of generality, we may assume $\varphi\left(\mathbf{G}, \overline{\mathbf{b}}_{i+1}\right)^{2} \subset \varphi\left(\mathbf{G}, \overline{\mathbf{b}}_{i}\right)$ for every $i \in \omega$. Let $\mathfrak{\mathfrak { A }}$ be a subset of $A u t(\mathbf{G} / A)$ containing exactly one extension of every $\sigma \in \mathfrak{A}$. Let $n=V C\left(\varphi^{*}(x, \bar{y})\right)$, let $\tau_{A}\left(\left(\bar{y}_{0, p}, \bar{y}_{1, p}, \ldots, \bar{y}_{n, p}\right)_{p \in \omega}\right)$ be a partial type over $A$ stating that the sequences $\left(\bar{y}_{k, p}\right)_{p \in \omega}$ have the same type over $A$ for every $k \in\{0, \ldots, n\}$ and let

$$
\begin{aligned}
& \rho_{\mathfrak{A}}\left(\left(\bar{y}_{0, p}, \bar{y}_{1, p}, \ldots, \bar{y}_{n, p}\right)_{p \in \omega}\right)=\tau_{A}\left(\left(\bar{y}_{0, p}, \bar{y}_{1, p}, \ldots, \bar{y}_{n, p}\right)_{p \in \omega}\right) \cup \\
& \left\{\varphi\left(x, \bar{y}_{1,0}\right) \wedge \cdots \wedge \varphi\left(x, \bar{y}_{n, 0}\right) \subset_{x} \varphi\left(x, \boldsymbol{\sigma} \overline{\mathbf{b}}_{i}\right), \varphi\left(k, \bar{y}_{1,0}\right) \wedge \cdots \wedge \varphi\left(k, \bar{y}_{n, 0}\right),\right. \\
& \varphi\left(x, \bar{y}_{0, p}\right) \subset_{x} \varphi\left(x, \overline{\mathbf{b}}_{i}\right), \varphi\left(x, \bar{y}_{0, p+1}\right)^{2} \subset_{x} \varphi\left(x, \bar{y}_{0, p}\right), \\
& \left.\varphi\left(h, y_{0, p}\right): i \in \omega, p \in \omega, h \in H, k \in H^{\mathfrak{A}}, \boldsymbol{\sigma} \in \overline{\mathfrak{A}}\right\} .
\end{aligned}
$$


We claim that $\rho_{\mathfrak{A}}$ is finitely satisfiable. For every $m \geqslant 1$, every $i_{1}, \ldots, i_{m} \in \omega$ and every $\boldsymbol{\sigma}_{1}, \ldots, \boldsymbol{\sigma}_{m}$ in $\overline{\mathfrak{A}}$, putting $i=\max \left\{i_{1}, \ldots, i_{m}\right\}+1$, one has

$$
\varphi\left(\mathbf{G}, \boldsymbol{\sigma}_{1} \overline{\mathbf{b}}_{i}\right) \cap \cdots \cap \varphi\left(\mathbf{G}, \boldsymbol{\sigma}_{m} \overline{\mathbf{b}}_{i}\right) \subset \varphi\left(\mathbf{G}, \boldsymbol{\sigma}_{1} \overline{\mathbf{b}}_{i_{1}}\right) \cap \cdots \cap \varphi\left(\mathbf{G}, \boldsymbol{\sigma}_{m} \overline{\mathbf{b}}_{i_{n}}\right) .
$$

By Lemma 2.2, there are $j>i$ and $j_{1}, \ldots, j_{n} \in\left\{i_{1}, \ldots, i_{m}\right\}$ with

$$
\varphi\left(\mathbf{G}, \boldsymbol{\sigma}_{j_{1}} \overline{\mathbf{b}}_{j}\right) \cap \cdots \cap \varphi\left(\mathbf{G}, \boldsymbol{\sigma}_{j_{n}} \overline{\mathbf{b}}_{j}\right) \subset \varphi\left(\mathbf{G}, \boldsymbol{\sigma}_{1} \overline{\mathbf{b}}_{i}\right) \cap \cdots \cap \varphi\left(\mathbf{G}, \boldsymbol{\sigma}_{m} \overline{\mathbf{b}}_{i}\right) .
$$

Putting $\left(\bar{c}_{1, p}, \ldots, \bar{c}_{n, p}\right)=\left(\boldsymbol{\sigma}_{j_{1}} \overline{\mathbf{b}}_{j+p}, \ldots, \boldsymbol{\sigma}_{j_{n}} \overline{\mathbf{b}}_{j+p}\right)$ for every $p$, one has

$$
\left(\bar{c}_{k, 0}, \bar{c}_{k, 1}, \ldots, \bar{c}_{k, p}\right)=\boldsymbol{\sigma}_{j_{k}}\left(\overline{\mathbf{b}}_{j}, \overline{\mathbf{b}}_{j+1}, \ldots, \overline{\mathbf{b}}_{j+p}\right) \text { for every } k \in\{1, \ldots, n\} .
$$

It follows that $\rho_{\mathfrak{A}}$ is consistent. Let $\left(\left(\overline{\mathbf{c}}_{0, p}, \overline{\mathbf{c}}_{1, p}, \ldots, \overline{\mathbf{c}}_{n, p}\right)_{p \in \omega}\right)$ be a realisation of $\rho_{\mathfrak{A}}$. As the sequences $\left(\overline{\mathbf{c}}_{k, p}\right)_{p \in \omega}$ have the same type over $A$ for every $k \in\{0, \ldots, n\}$, there are $\boldsymbol{\sigma}_{1}, \ldots, \boldsymbol{\sigma}_{n}$ in $\operatorname{Aut}(\mathbf{G} / A)$ such that $\boldsymbol{\sigma}_{k}\left(\overline{\mathbf{c}}_{0, p}\right)_{p \in \omega}=\left(\overline{\mathbf{c}}_{k, p}\right)_{p \in \omega}$ for every $k \in\{1, \ldots, n\}$. Putting $\mathbf{K}=$ $\bigcap_{p \in \omega} \varphi\left(\mathbf{G}, \overline{\mathbf{c}}_{0, p}\right)$, one has $H=\mathbf{K} \cap G$ and $\bigcap_{\sigma \in \mathfrak{A}} H^{\sigma}=\mathbf{K}^{\sigma_{1}} \cap \cdots \cap \mathbf{K}^{\sigma_{n}} \cap G$. Considering the union of the types $\rho_{\mathfrak{A}}\left(\left(\bar{y}_{0, p}, \bar{y}_{1, p, \mathfrak{A}}, \ldots, \bar{y}_{n, p, \mathfrak{A}}\right)_{p \in \omega}\right)$ when $A$ and $\mathfrak{A}$ vary, one can find a group $\mathbf{K}$ that depends neither on $A$ nor on $\mathfrak{A}$.

(3) By Lemma 3.4, we may assume that $\mathbf{H}$ is defined by a countable type. By Theorem 2.10 applied in $\mathbf{G}$ to $H \subset \mathbf{H}$ and $N_{G}(H)$, there are two nice subgroups $\mathbf{K}, \mathbf{N} \subset G$ such that $H \subset \mathbf{K} \subset \mathbf{H}$ and $N_{G}(H) \subset \mathbf{N} \subset \mathbf{N}_{\mathbf{G}}(\mathbf{K})$. One thus has $H=\mathbf{K} \cap G$ and $N_{G}(H)=\mathbf{N} \cap G$.

\section{EnVELOPES IN A DEFinABLE GROUP}

Let us recall the following results from [She09] and [dA13].

Theorem 4.1. Let $\mathfrak{C}$ be a monster model of a NIP theory and $G$ a group definable in $\mathfrak{C}$.

(1) (S. Shelah) If $G$ has an infinite abelian subgroup $A$, then it has a definable abelian subgroup that contains infinitely many elements of $A$.

(2) (R. de Aldama) If $G$ has a nilpotent subgroup $N$ of class $n$ with $|N|<|\mathfrak{C}|$, then it has a definable nilpotent subgroup of class $n$ that contains $N$.

(3) (R. de Aldama) If $G$ has a normal soluble subgroup $S$ of derived length $\ell$ with $|S|<|\mathfrak{C}|$, then it has a definable soluble subgroup of derived length $\ell$ that contains $S$.

Throughout the section, we consider a NIP group $G$ and $\mathbf{G}$ a $|G|^{+}$-saturated elementary extension.

Theorem 4.2 (abelian envelope). Let $A \subset G$ be an abelian subgroup. There is an external subgroup $H=\mathbf{H} \cap G$ with $A \subset H$ such that $\mathbf{H}$ is abelian, $H$ is $A$-invariant and normalised by $N_{G}(A)$.

First proof (Adapted from [dA13, Lemma 2.1]). For any subset $B \subset \mathbf{G}$, we put

$$
C(B)=\bigcap_{\substack{b \in B \\[b, A]=1}} C_{\mathbf{G}}(b) .
$$

We claim that there is a finite subset $B \subset \mathbf{G}$ such that $C(A \cup B)$ is abelian. Otherwise we construct by induction on $n$ two sequences $\left(a_{n}\right)_{n \geqslant 1}$ and $\left(b_{n}\right)_{n \geqslant 1}$ such that for every $n$, both $a_{n}$ and $b_{n}$ belong to $C\left(A \cup\left\{a_{k}, b_{k}: k<n\right\}\right)$ and $\left[a_{n}, b_{n}\right] \neq 1$. It follows that $\left[a_{i}, b_{j}\right]=1$ if 
and only if $i \neq j$, so that the family $\left(C_{\mathbf{G}}\left(a_{i}\right)\right)_{i \geqslant 1}$ does not satisfy the Baldwin-Saxl chain condition, a contradiction. As $\mathbf{G}$ is $|A|^{+}$-saturated, by the Compactness Theorem, there are $c_{1}, \ldots, c_{n}$ in $B$ such that $C_{\mathbf{G}}\left(c_{1}, \ldots, c_{n}\right)$ is abelian, and contains $A$. By Lemma 3.5, the group

$$
\bigcap_{g \in N_{G}(A)} \bigcap_{\sigma \in \operatorname{Aut}(G / A)} C_{G}\left(\sigma\left(c_{1}^{g}\right), \ldots, \sigma\left(c_{n}^{g}\right)\right)
$$

is external.

Second proof. $Z\left(C_{G}(A)\right)$ is external (as an abelian group) by Theorem 3.7.1 and 3.7.2. It is also $A$-invariant and normalised by $N_{G}(A)$.

Remark 4.3. The simpler second proof provides an abelian envelope defined by the formula $Z\left(C_{G}\left(x_{1}, \ldots, x_{n}\right)\right)$, whereas the first proof provides an envelope defined by the simpler formula $C_{G}\left(x_{1}, \ldots, x_{n}\right)$.

Theorem 4.4 (soluble envelope 1). Let $S \subset G$ be a soluble subgroup of derived length $\ell$. There is a discernible subgroup $H=\mathbf{H} \cap G$ with $S \subset H$ such that $\mathbf{H}$ is soluble of derived length $\ell, H$ is $S$-invariant and normalised by $N_{G}(S)$.

Proof. By induction on $\ell$, we show that there are two nice subgroups $\mathbf{H}, \mathbf{N} \subset \mathbf{G}$ defined by countable partial types, such that $\mathbf{H}$ is soluble of derived length $\ell$,

$$
S \subset \mathbf{H}, \quad N_{G}(S) \subset \mathbf{N}, \quad \text { and } \quad \mathbf{H} \triangleleft \mathbf{N} .
$$

If $\ell=0$, there is nothing to show. If the result holds for every $\ell$-soluble subgroup of $G$ and if $S$ is soluble of derived length $\ell+1$, there are nice subgroups $\mathbf{K}, \mathbf{M} \subset \mathbf{G}$ defined by countable partial types, such that $\mathbf{K}$ is soluble of derived length $\ell, S^{\prime} \subset \mathbf{K}, N_{G}\left(S^{\prime}\right) \subset \mathbf{M}$ and $\mathbf{K} \triangleleft \mathbf{M}$. As $N_{G}(S) \subset N_{G}\left(S^{\prime}\right)$, one has $N_{G}(S) \subset \mathbf{M}$. We thus have

$$
S \mathbf{K} / \mathbf{K} \subset \mathbf{M} / \mathbf{K} \text {. }
$$

As $s \mathbf{K}=\mathbf{K} s$ holds for every $s$ in $S$, one has $[S \mathbf{K}, S \mathbf{K}] \subset S^{\prime} \mathbf{K} \subset \mathbf{K}$, hence $(S \mathbf{K})^{\prime} \subset \mathbf{K}$, so the group $S \mathbf{K} / \mathbf{K}$ is abelian.

By Corollary 1.9, the pure group $\mathbf{M} / \mathbf{K}$ is a NIP structure, so the formula $y x=x y$ does not have the independence property in $\mathbf{M} / \mathbf{K}$. As $\mathbf{K}$ and $\mathbf{M}$ are defined by countable types and as $\mathbf{G}$ is $|S|^{+}$-saturated, $\mathbf{M} / \mathbf{K}$ is also $|S|^{+}$-saturated. By Theorem 4.2 , there are $\mathbf{a}_{1}, \ldots, \mathbf{a}_{n}$ in $\mathbf{M}$ such that $C_{\mathbf{M} / \mathbf{K}}\left(\mathbf{a}_{1} \mathbf{K}, \ldots, \mathbf{a}_{n} \mathbf{K}\right)$ is abelian and contains $S \mathbf{K} / \mathbf{K}$. It follows that the group

$$
\mathbf{L}=\bigcap_{i=1}^{n}\left\{x \in \mathbf{M}:\left[x, \mathbf{a}_{i}\right] \subset \mathbf{K}\right\}
$$

is nice, soluble of derived length $\ell+1$ and contains $S$. By Theorem 2.10 applied in $\mathbf{G}$ to $S \subset \mathbf{L}$ and $N_{G}(S)$, there are nice subgroups $\mathbf{H}, \mathbf{N} \subset G$ with $S \subset \mathbf{H} \subset \mathbf{L}, N_{G}(S) \subset \mathbf{N}$ and $\mathbf{H} \triangleleft \mathbf{N}$. This ends the induction.

Putting $H=\mathbf{H} \cap G$, the subgroup

$$
\bigcap_{\sigma \in \operatorname{Aut}(G / S)} \sigma(H)
$$

is discernible by Theorem 3.8.2, $S$-invariant and normalised by $N_{G}(S)$. 
Corollary 4.5 (soluble envelope 2). Let $S \subset G$ be a soluble subgroup of derived length $\ell$. There is an externally definable subgroup $X=\mathbf{X} \cap G$ of $G$ such that $S \subset X$ and $\langle\mathbf{X}\rangle$ is a soluble subgroup of $\mathbf{G}$ of derived length $\ell$.

Proof. By Zorn's Lemma, we may assume that $S$ is a maximal soluble subgroup of $G$ of derived length $\ell$. By Theorem 4.4, there is a formula $\varphi(x, y)$ and a subset $\mathbf{A} \subset \mathbf{G}$ such that $\bigcap\{\varphi(\mathbf{G}, a): a \in \mathbf{A}\}$ defines a nice soluble subgroup of $\mathbf{G}$ containing $S$. We write $\varphi(\mathbf{G}, a)^{n}$ for the set of products of $n$ elements of $\varphi(\mathbf{G}, a)$. We say that a subset $\mathbf{X} \subset \mathbf{G}$ is soluble of derived length $\ell$ if $\mathbf{X}$ satisfies all the commutator identities satisfied by a soluble group of derived length $\ell$. For a definable set $\mathbf{X}$, being soluble of derived length $\ell$ is a first order property. The partial type over $S$

$$
\pi(x)=\left\{\varphi(s, x), \varphi(\mathbf{G}, x)^{n} \text { is soluble of derived length } \ell: s \in S, n \in \omega\right\}
$$

is finitely satisfiable in $\mathbf{A}$. Let $\mathbf{s} \in \mathbf{G}$ be a realisation of $\pi$ and let $\mathbf{X}=\varphi(\mathbf{G}, \mathbf{s})$. The set $\mathbf{X} \cap G$ is a subgroup by maximality of $S$.

Remark 4.6. In an arbitrary group $G$, if a subset $X \subset G$ satisfies all the commutator identities satisfied by a nilpotent group of class $n$, we call $X$ a nilpotent subset of class $n$. If a $X$ is nilpotent of class $n$, then $X$ generates a nilpotent subgroup of class $n$. If $X$ is in addition definable, then it is contained in a definable nilpotent subgroup of class $n$. This can be shown taking $Z\left(C_{G}(X)\right)$ for $n=1$, and $Z_{n}\left(E_{n}\right)$ for arbitrary $n$, with $E_{n}$ defined by induction putting $E_{0}=G$ and $E_{k+1}=\left\{x \in E_{k}:\left[x, C_{E_{k}}^{k+1}(X)\right] \subset C_{E_{k}}^{k}(X)\right\}$ (see [AB14]). However, if $X$ is merely soluble of derived length 2 , then $X$ may not even generate a soluble subgroup. Consider for instance two generators $a$ and $b$ of the alternating group $A_{5}$. The set $\{a, b\}$ obviously satisfies the equation $[[x, y],[z, t]]=1$, but $A_{5}$ is not solvable.

Theorem 4.7 (normal soluble envelope). Let $S \subset G$ be a normal soluble subgroup of derived length $\ell$. There is a normal, soluble of derived length $\ell$, definable subgroup $\mathbf{H} \subset \mathbf{G}$ such that $S \subset \mathbf{H}$.

Proof. Note that $S$ need not be normal in $\mathbf{G}$, so Theorem 4.1.3 does not apply. The following proof is due to $\mathrm{F}$. Wagner. We show that the result holds for every $G$ and every $\ell$-soluble subgroup of $G$ by induction on $\ell$. For $\ell=1$, the group $S$ is abelian and normal. For any elementary extension $\mathbf{G}$ of $G$ and $s_{1}, \ldots, s_{n}$ in $S$, the conjugacy classes $s_{1}^{\mathbf{G}}, \ldots, s_{n}^{\mathbf{G}}$ generate an abelian subgroup of $\mathbf{G}$. By the Baldwin Saxl chain condition, there is a natural number $n$ such that the partial type over $S$

$$
\pi\left(x_{1}, \ldots, x_{n}\right)=\left\{s \in C_{G}\left(x_{1}^{G}, \ldots, x_{n}^{G}\right), C_{G}\left(x_{1}^{G}, \ldots, x_{n}^{G}\right) \subset C_{G}(s): s \in S\right\}
$$

is finitely satisfiable in $S$. As $\mathbf{G}$ is $|S|^{+}$-saturated, there are $\mathbf{a}_{1}, \ldots, \mathbf{a}_{n}$ in $\mathbf{G}$ such that

$$
S \subset C_{\mathbf{G}}\left(\mathbf{a}_{1}^{\mathbf{G}}, \ldots, \mathbf{a}_{n}^{\mathbf{G}}\right) \subset C_{\mathbf{G}}(S) .
$$

It follows that $Z\left(C_{\mathbf{G}}\left(\mathbf{a}_{1}^{\mathbf{G}}, \ldots, \mathbf{a}_{n}^{\mathbf{G}}\right)\right)$ is normal in $\mathbf{G}$, abelian and contains $S$. If $S$ is soluble of derived length $\ell+1$, by induction hypothesis, $S^{\prime}$ is contained in a normal, soluble of derived length $\ell$, definable subgroup $\mathbf{K}$ of $\mathbf{G}$. $S \mathbf{K} / \mathbf{K}$ is a normal abelian subgroup of $\mathbf{G} / \mathbf{K}$. As $\mathbf{G} / \mathbf{K}$ is interpretable in $M$, it is a NIP pure group. As $\mathbf{G} / \mathbf{K}$ is $|S|^{+}$-saturated, $S \mathbf{K} / \mathbf{K}$ is contained in a normal abelian interpretable subgroup $\mathbf{H} / \mathbf{K}$ of $\mathbf{G} / \mathbf{K}$, and $\mathbf{H}$ is as desired. 


\section{Further chain CONDitions à la Baldwin SAXL}

We consider a NIP structure $M$ and a type definable group $G \subset M^{m}$. Two new difficulties appear: $G$ need not be the intersection of definable groups, and if $H \subset G$ is a type definable normal subgroup, the pure group $G / H$ might have the independence property unless the formulas defining $H$ relatively to $G$ are controlled.

5.1. Relatively nice subgroups. We say that $G \subset M^{m}$ is a type definable group in $M$ if there is a definable map $*$ from $M^{2 m}$ to $M^{m}$, a partial $m$-type $\pi(\bar{x})$ with parameters in $M$ such that $G=\pi(M)$ and, for every elementary extension $\mathbf{M}$ of $M$, the subset $\pi(\mathbf{M}) \subset \mathbf{M}^{m}$ is a group for $*^{\mathbf{M}}$. We call $\pi$ a defining type of $G$. Assuming $*$ to be definable rather than type definable is no restriction by a compactness argument (see [Poi85, page 170]).

By the Compactness Theorem, there exists a sequence $\beta$ of definable subsets $\beta_{i} \subset M^{n}$ that contain $G$, and a definable involution ${ }^{-1}$ from $\beta_{0}$ to $\beta_{0}$ with the following properties: for every $\bar{x} \in \beta_{0}$, one has $\bar{x} \bar{x}^{-1}=\bar{x}^{-1} \bar{x}=1$ and $\bar{x} 1=1 \bar{x}=\bar{x}$; for every $n \in \omega$ one has $\beta_{n}^{-1}=\beta_{n}$ and $\beta_{n+1} * \beta_{n+1} \subset \beta_{n}$; for every $\bar{x}_{1}, \ldots, \bar{x}_{2^{n}}$ in $\beta_{n}$, the element $\bar{x}_{1} \bar{x}_{2} \cdots \bar{x}_{2^{n}}$ is well-defined, independently of the order of the $2^{n}-1$ computations. We call $\beta$ a base of $G$.

For any subset $X \subset \beta_{n}$ and $k \leqslant 2^{n}$, we write $X^{k}$ for the subset of $\beta_{0}$ consisting of products $x_{1} x_{2} \cdots x_{k}$ of any $k$ elements $x_{1}, \ldots, x_{k}$ of $X$, and $X^{\times k}$ for the Cartesian product $X \times \cdots \times X$. For a set $Y$, when there is no ambiguity, we go on writing $Y^{k}$ for the Cartesian product $Y \times \cdots \times Y$. A subset $X \subset \beta_{0}$ such that $1 \in X$ and $X^{-1}=X$ is called symmetric.

Throughout the section, we consider $G=\pi(M)$ a type definable group in $M$ of base $\beta$. A type definable subgroup $H \subset G$ is called relatively definable in $G$ if there is a formula $\varphi(\bar{x})$ such that $\pi \cup\{\varphi\}$ is a defining type of $H$. We call $\varphi$ a defining formula of $H$. More generally:

Definition 5.1 (relatively nice subgroup). A type definable subgroup $H \subset G$ is relatively nice in $G$ if there is a formula $\varphi(\bar{x}, \bar{y})$ and a subset $A \subset M^{k}$ such that $\pi(\bar{x}) \cup\{\varphi(\bar{x}, \bar{a}): \bar{a} \in A\}$ is a defining type of $H$, the family $\{\pi(\mathbf{M}) \cap \varphi(\mathbf{M}, \bar{a}): \bar{a} \in A\}$ is a filter for every elementary extension $\mathbf{M}$ of $M$ and the sets $\varphi(G, \bar{a}) \subset \beta_{0}$ are symmetric for every $\bar{a} \in A$. We call $\varphi(\bar{x}, \bar{y})$ a defining formula of $H$.

A family $\mathfrak{H}$ of relatively nice subgroups of $G$ is called uniform if its members have a common defining formula.

Lemma 5.2 (Baldwin Saxl chain condition for relatively nice subgroups). If $\mathfrak{H}$ is a uniform family of relatively nice subgroups of $G$, there is $n \in \omega$ such that any finite intersection of members of $\mathfrak{H}$ is the intersection of at most $n$ of them.

Proof. Otherwise, by the usual Baldwin Saxl argument, for every $n \in \omega$ one would find $H_{1}, \ldots, H_{n}$ in $\mathfrak{H}$ and tuples $\left(b_{J}\right)_{J \subset\{1, \ldots, n\}}$ of elements in $G$ such that $b_{J} \in H_{i} \Longleftrightarrow i \in J$. Let $\varphi(\bar{x}, \bar{y})$ be a common defining formula for the members of $\mathfrak{H}$, and let $\pi(\bar{x}) \cup\left\{\varphi\left(\bar{x}, \bar{a}_{i}\right): \bar{a}_{i} \in A_{i}\right\}$ a defining type for $H_{i}$. Let $B_{J}$ and $B_{i}$ be the finite sets

$$
B_{J}=\left\{b_{J}: J \subset\{1, \ldots, n\}\right\}, \quad B_{i}=\left\{b_{J} \in B: i \in J\right\} .
$$


As the family $\left\{\varphi\left(B_{J}, \bar{a}_{i}\right): \bar{a}_{i} \in A_{i}\right\}$ is a filter for every $i \in\{1, \ldots, n\}$, by the Compactness Theorem, there are $\left(\overline{\mathbf{a}}_{1}, \ldots, \overline{\mathbf{a}}_{n}\right)$ in $\mathbf{G}$ such that $B_{i} \subset \varphi\left(B_{J}, \overline{\mathbf{a}}_{i}\right) \subset \varphi\left(B_{J}, \bar{a}_{i}\right)$ for every $i$ and every $\bar{a}_{i} \in A_{i}$, so that $\mathbf{M}=\varphi\left(b_{J}, \overline{\mathbf{a}}_{i}\right)$ if and only if $i \in J$, a contradiction.

Corollary 5.3. If $\mathfrak{H}$ is a uniform family of relatively nice subgroups of $G$, then $\bigcap_{H \in \mathfrak{H}} H$ is relatively nice and the family $\left\{\bigcap_{H \in \mathfrak{K}} H: \mathfrak{K} \subset \mathfrak{H}\right\}$ is uniform.

Proof. Let $n$ be the natural number provided by Lemma 5.2 and for every $H$ in $\mathfrak{H}$, let $\left\{\varphi(\bar{x}, \bar{a}): \bar{a} \in A_{H}\right\}$ be a type defining $H$ relatively to $G$. Let $A$ be the set $\bigcup_{H \in \mathfrak{H}} A_{H}$. Calling $\psi(\bar{x}, \bar{y})$ the formula $\varphi\left(\bar{x}, \bar{y}_{1}\right) \wedge \cdots \wedge \varphi\left(\bar{x}, \bar{y}_{n}\right)$, the type $\left\{\psi(\bar{x}, \bar{a}): \bar{a} \in A^{n}\right\}$ defines the group $\bigcap_{H \in \mathfrak{H}} H$ and the family $\left\{\pi(\mathbf{M}) \cap \psi(\mathbf{M}, \bar{a}): \bar{a} \in A^{n}\right\}$ is a filter by Lemma 5.2 .

5.2. Uniform definability. A third difficulty arises. If $H \subset G$ is a relatively nice subgroup with defining type $\left\{\varphi\left(\bar{x}, \bar{a}_{i}\right): i \in \omega\right\}$ and $\left|\bar{a}_{i}\right|=k$, it is not clear whether there is a uniform partial type $\rho\left(\left(\bar{y}_{i}\right)_{i \in \omega}\right)$ such that for every countable $B \subset M^{k}, M \models \rho(B)$ implies that $\bigcap_{\bar{b} \in B} \varphi(G, \bar{b})$ is a relatively nice subgroup of $G$. This prevents applying compactness arguments. We introduce therefore a strengthening of the preceding notions. The Compactness Theorem ensures that for all $i \in \omega$, there is $j \in \omega$ and a definable set $X_{i}$ with $G \subset X_{i} \subset \beta_{1}$ and

$$
\left(X_{i} \cap \varphi\left(M, a_{j}\right)\right)^{2} \subset \varphi\left(M, a_{i}\right) .
$$

Definition 5.4 (nice subgroup). We say that $H \subset G$ is nice (in $G$ ) if it is relatively nice with defining type $\{\varphi(\bar{x}, \bar{a}): \bar{a} \in A\}$ and there is a definable set $X$ with $G \subset X \subset \beta_{1}$ such that for all $\bar{a} \in A$, there is $\bar{b} \in A$ such that

$$
(X \cap \varphi(M, \bar{b}))^{2} \subset \varphi(M, \bar{a}) .
$$

We call $X$ a second base for $N$.

If $H \subset G$ is nice in $G$ and $K \subset H$ is nice in $H$, then $K$ is nice in $G$.

Definition 5.5 (uniform family of nice subgroups). A family $\mathfrak{H}$ of nice subgroups of $G$ is uniform if its members have the same defining formula and share a common second base.

Lemma 5.6 (a uniform family is closed under intersections). If $\mathfrak{H}$ is a uniform family of nice subgroups of $G$, then $\bigcap_{H \in \mathfrak{H}} H$ is nice in $G$, and the family $\left\{\bigcap_{H \in \mathfrak{K}} H: \mathfrak{K} \subset \mathfrak{H}\right\}$ is uniform.

Proof. By Corollary 5.2, the subgroup $\bigcap_{H \in \mathfrak{H}} H$ is relatively nice in $G$, and it is easy to see that a common second base for $\mathfrak{H}$ is a common second base for the family $\left\{\bigcap_{H \in \mathfrak{K}} H: \mathfrak{K} \subset \mathfrak{H}\right\}$.

Example 5.7. A relatively definable subgroup $H=\varphi(G)$ is nice. As $(G \cap \varphi(M))^{2} \subset \varphi(M)$, by the Compactness theorem, $H$ has a second base.

Example 5.8. For any $g \in G$, the centraliser $C_{G}(g)$ is relatively definable in $G$, and the family $\left\{C_{G}(g): g \in G\right\}$ is uniform, sharing $\beta_{2}$ as a second base if one puts $\beta_{1}=\phi_{1}(M)$ and chooses $\phi_{1}(\bar{x}) \wedge \bar{x} \bar{y}=\bar{y} \bar{x}$ as a defining formula. In particular, $C_{G}(A)$ is nice for any $A \subset G$. 
Example 5.9. If $G$ is type definable over $A \subset M$, if $\mathfrak{A} \subset A u t(M / A)$ and $H \subset G$ is nice with an $A$-definable second base $X$, then $\left\{H^{\sigma}: \sigma \in \mathfrak{A}\right\}$ shares $X$ as a second base, and $\bigcap_{\sigma \in \mathfrak{A}} H^{\sigma}$ is nice.

Example 5.10. Let $G$ be type definable over $A$, and $H \subset G$ a relatively nice subgroup with defining type $\left\{\varphi\left(x, a_{i}\right): i \in I\right\}$ where $I$ is a linearly ordered set. If $\left(a_{i}\right)_{i \in I}$ is indiscernible over $A$, the set $X_{i}$ provided by (2) does not depend on $a_{i}$, so $H$ is nice.

Lemma 5.11. If $H \subset G$ is a nice subgroup and $g \in G$, then $H^{g}$ is nice and the family $\left\{H^{g}: g \in G\right\}$ is uniform. In particular $H^{A}$ is nice for any $A \subset G$.

Proof. By Lemma 5.6, we need only find a common second base for $\left\{H^{g}: g \in G\right\}$. Let $X \subset \beta_{1}$ be a second base for $H$ and $\{\varphi(\bar{x}, \bar{a}): \bar{a} \in A\}$ a defining type. For all $\bar{a} \in A$ there is $\bar{b} \in A$ such that

$$
(X \cap \varphi(M, \bar{b}))^{2} \subset \varphi(M, \bar{a}) .
$$

By the Compactness theorem, there is a definable $Y \subset \beta_{3}$ such that $G \subset Y^{3} \subset X$. It follows that for every $g \in G$, one has

$$
\left(Y \cap \varphi(M, \bar{b})^{g}\right)^{2} \subset \varphi(M, \bar{a})^{g} .
$$

Lemma 5.12 (Baldwin Saxl chain condition for subsets). Let $\mathfrak{X}$ be a family of subsets of $\beta_{0}$. For all $X \in \mathfrak{X}$, let $X^{1 / 3} \subset \beta_{2}$ be symmetric with $\left(X^{1 / 3}\right)^{3} \subset X$ and let $\mathfrak{X}^{1 / 3}=\left\{X^{1 / 3}: X \in \mathfrak{X}\right\}$ be uniformly definable by a formula $\varphi(\bar{x}, \bar{y})$. Let $n=V C\left(\varphi^{*}(\bar{x}, \bar{y})\right)$. For every $X \in \mathfrak{X}$, let $X^{1 / 3 n} \subset \beta_{n+1}$ be symmetric with $\left(X^{1 / 3 n}\right)^{n} \subset X^{1 / 3}$. For all $X_{1}, \ldots, X_{n+1} \in \mathfrak{X}$, there is $j \in\{1, \ldots, n+1\}$ with

$$
X_{1}^{1 / 3 n} \cap \cdots \cap X_{j-1}^{1 / 3 n} \cap X_{j+1}^{1 / 3 n} \cap \cdots \cap X_{n+1}^{1 / 3 n} \subset X_{1} \cap \cdots \cap X_{n+1} .
$$

Proof. Similar to the proof of Lemma 2.2.

Corollary 5.13 (uniform definability of niceness). Let $H \subset G$ be a type definable subgroup with defining type $\{\varphi(\bar{x}, \bar{a}): \bar{a} \in A\} . H$ is nice if and only if there is a definable $X$ with $G \subset X \subset \beta_{1}$ such that for all $\bar{a} \in A$ there is $\bar{b} \in A$ with

$$
(X \cap \varphi(M, \bar{b}))^{2} \subset \varphi(M, \bar{a}) .
$$

Proof. Without loss of generality, we put $\beta_{0}=\phi(M)$, replace $\varphi(\bar{x}, \bar{y})$ by $\phi_{0}(\bar{x}) \wedge \varphi(\bar{x}, \bar{y}) \wedge$ $\varphi\left(\bar{x}^{-1}, \bar{y}\right)$ and assume that $X=\beta_{1}$. Let $\mathfrak{X}=\{\varphi(M, \bar{a}): \bar{a} \in A\}$ and $\mathfrak{X}^{1 / 3 j}=\left\{\beta_{j+1} \cap \varphi(M, \bar{a})\right.$ : $\bar{a} \in A\}$ for any non-zero $j \in \omega$. Let $\bar{a} \in A$. By assumption, there are $\bar{a}=\bar{b}_{0}, \bar{b}_{1}, \ldots, \bar{b}_{j}$ such that for all $\ell \in\{0, \ldots, j\}$,

$$
\left(\beta_{1} \cap \varphi\left(M, \bar{b}_{\ell+1}\right)\right)^{2} \subset \varphi\left(M, \bar{b}_{\ell}\right)
$$

It follows that

$$
\left(\beta_{j+1} \cap \varphi\left(M, \bar{b}_{j}\right)\right)^{j} \subset\left(\beta_{j+1} \cap \varphi\left(M, \bar{b}_{j}\right)\right)^{2^{j}} \subset\left(\beta_{j} \cap \varphi\left(M, \bar{b}_{j-1}\right)\right)^{2^{j-1}} \subset \cdots \subset \beta_{1} \cap \varphi\left(M, \bar{b}_{0}\right) .
$$

Putting $n=V C\left(\varphi^{*}(\bar{x}, \bar{y})\right)$, the families $\mathfrak{X}, \mathfrak{X}^{1 / 3}$ and $\mathfrak{X}^{1 / 3 n}$ satisfy the assumptions of Lemma 5.12, so the family $\left\{\beta_{n+2} \cap \bigcap_{\bar{a} \in B} \varphi(M, \bar{a}): \bar{a} \in B,|B| \leqslant n+1\right\}$ is a filter. 
5.3. Normaliser. Let $H \subset G$ be a nice subgroup with defining type $\{\varphi(\bar{x}, \bar{a}): \bar{a} \in A\}$ and $N \subset G$ a type definable subgroup that normalises $H$. By the Compactness Theorem, for all $\bar{a} \in A$, there are $\bar{b} \in A$, definable sets $X_{\bar{a}}, K_{\bar{a}}$ with $G \subset X_{\bar{a}} \subset \beta_{2}$ and $N \subset K_{\bar{a}} \subset \beta_{2}$ such that for all $g \in K_{\bar{a}}$,

$$
\left(X_{\bar{a}} \cap \varphi(M, \bar{b})\right)^{g} \subset \varphi(M, \bar{a}) .
$$

Definition 5.14 (uniformly normal subgroup). If $H \subset G$ is nice and normalised by a type definable subgroup $N \subset G$, we say that $H$ is uniformly normalised by $N$ if there are definable sets $X, K$ with $G \subset X \subset \beta_{2}$ and $N \subset K \subset \beta_{2}$ such that for all $\bar{a} \in A$, there is $\bar{b} \in A$ such that for all $g \in K$,

$$
(X \cap \varphi(M, \bar{b}))^{g} \subset \varphi(M, \bar{a}) .
$$

Theorem 5.15 (normalising a nice envelope). Let $M$ be $\kappa$-saturated for $\kappa>\aleph_{0}$ and let $H \subset G$ be a nice subgroup (resp. relatively definable) defined by a partial type of size $<\kappa$. Let $A \subset H$ and $N_{A} \subset N_{G}(A)$ be subgroups of cardinality $<\kappa$. There are a nice (resp. a conjunction of a uniform family of relatively definable) subgroup $E \subset G$, and a type definable subgroup $N_{E} \subset G$ such that

$$
A \subset E \subset H \quad \text { and } \quad N_{A} \subset N_{E} \subset N_{G}(E) .
$$

Moreover, $N_{E}$ normalises E uniformly.

Proof. Let $\{\varphi(x, \bar{b}): \bar{b} \in B\}$ be a defining type for $H$. There is a definable $X_{0} \subset \beta_{1}$ containing $G$ such that for all $\bar{b} \in B$, there is $\bar{c} \in B$ with

$$
\left(\varphi(M, \bar{c}) \cap X_{0}\right)^{2} \subset \varphi(M, \bar{b}) .
$$

There are also definable symmetric $X_{n} \subset \beta_{n+1}$ such that $X_{n+1}^{2} \subset X_{n}$ for all $n \in \omega$. As $G$ is the intersection of type definable groups defined by such countable types, we may assume without loss of generality that $G=\bigcap_{n \in \omega} X_{n}$. By Lemma 5.12 and (3), replacing $\varphi(\bar{x}, \bar{y})$ by a finite conjunction of $\varphi\left(\bar{x}, \bar{y}_{i}\right)$, one may also assume that $\left\{\varphi(M, \bar{b}) \cap X_{0}: \bar{b} \in B\right\}$ is a filter.

Claim 2. There is $n \in \omega$ such that for all $\bar{b} \in B$ and $a_{1}, \ldots, a_{n} \in G$ with $A \subset \bigcap_{1 \leqslant i \leqslant n} \varphi(G, \bar{b})^{a_{i}}$, there are a nice subgroup $K \subset G$ with countable defining type $\{\varphi(\bar{x}, \bar{c}): \bar{c} \in C\}$, some $\alpha_{1}, \ldots, \alpha_{n} \in G$ and $\bar{c} \in C$ such that for all $g \in N_{A}$

$$
\left(\bigcap_{i=1}^{n} \varphi(M, \bar{c})^{\alpha_{i}} \cap X_{n}\right)^{g} \subset \bigcap_{i=1}^{n} \varphi(M, \bar{b})^{a_{i}}, \quad\left(\varphi(M, \bar{c}) \cap X_{0}\right)^{2} \subset \varphi(M, \bar{b}) \quad \text { and } \quad A \subset \bigcap_{i=1}^{n} K^{\alpha_{i}} \cap K .
$$

Proof of Claim 2. Fix $\bar{b} \in B$. By the Compactness Theorem, there is an upper bound $n \in \omega$ for $\left\{\operatorname{VC}\left(\varphi(\bar{y}, \bar{b})^{\bar{x}}\right): \bar{b} \in M\right\}$. By Lemma 5.11, the family $\left\{H^{g}: g \in G\right\}$ is uniform, of second base $X_{k}$ say, so there exists $\bar{b}_{1} \in B$ such that for all $g \in G$,

$$
\left(\varphi\left(M, \bar{b}_{1}\right)^{g} \cap X_{n+k}\right)^{3 n} \subset \varphi(M, \bar{b})^{g} \text { and }\left(\varphi\left(M, \bar{b}_{1}\right) \cap X_{0}\right)^{2} \subset \varphi(M, \bar{b}) .
$$

By Lemma 5.12, for every $g_{1}, \ldots, g_{n+1} \in G$, there is $i \in\{1, \ldots, n+1\}$ with

$$
\bigcap_{j \in\{1, \ldots, n+1\} \backslash\{i\}} \varphi\left(M, \bar{b}_{1}\right)^{g_{j}} \cap X_{n+k} \subset \bigcap_{j=1}^{n+1} \varphi(M, \bar{b})^{g_{j}} .
$$


By induction on $|J|$, for all finite $J \subset G$, there are $J_{n} \subset J$ with $\left|J_{n}\right|<n+1$ and $\bar{b}_{2} \in B$ with

$$
\bigcap_{g \in J_{n}} \varphi\left(M, \bar{b}_{2}\right)^{g} \cap X_{n+k} \subset \bigcap_{g \in J} \varphi(M, \bar{b})^{g} \quad \text { and } \quad\left(\varphi\left(M, \bar{b}_{2}\right) \cap X_{0}\right)^{2} \subset \varphi(M, \bar{b}) .
$$

We put $X_{i}=\phi_{i}(M)$ for every $i \in \omega$ and consider the partial type

$$
\begin{gathered}
\rho\left(\bar{x}_{1}, \ldots, \bar{x}_{n},\left(\bar{z}_{i}\right)_{i \in \omega}\right)=\left\{\left(\varphi\left(\bar{x}, \bar{z}_{i+1}\right) \wedge \phi_{0}(\bar{x})\right)^{2} \subset_{\bar{x}} \varphi\left(\bar{x}, \bar{z}_{i}\right), \varphi\left(\bar{a}, \bar{z}_{i}\right),\right. \\
\left(\varphi\left(\bar{x}, \bar{z}_{0}\right) \wedge \phi_{0}(\bar{x})\right)^{2} \subset_{\bar{x}} \varphi(\bar{x}, \bar{b}),\left(\bigwedge_{j=1}^{n} \varphi\left(\bar{x}, \bar{z}_{0}\right)^{\bar{x}_{j}} \wedge \phi_{n+k}(\bar{x})\right) \subset_{\bar{x}}\left(\bigwedge_{j=1}^{n} \varphi(\bar{x}, \bar{b})^{a_{j}}\right)^{g}, \\
\left.\varphi\left(\bar{a}, \bar{z}_{i}\right)^{a_{1}}, \ldots, \varphi\left(\bar{a}, \bar{z}_{i}\right)^{a_{n}}, \bar{x}_{1} \in G, \ldots, \bar{x}_{n} \in G: g \in N_{G}(A), \bar{a} \in A, i \in \omega\right\} .
\end{gathered}
$$

By (5), the type $\rho$ is finitely satisfiable in $N_{G}(A)^{\times n} \times B^{\omega}$. Let $\left(\alpha_{1}, \ldots, \alpha_{n}, C\right)$ be a realisation of $\rho$. By Corollary 5.13, the type $\{\varphi(\bar{x}, \bar{c}): \bar{c} \in C\}$ defines a nice subgroup of $G$.

Let $n \in \omega$ provided by Claim 2. We may assume that $n \geqslant 6$. We call $\psi\left(\bar{x}, \bar{y}_{1} \ldots, \bar{y}_{n+1}\right)$ the formula $\varphi\left(\bar{x}, \bar{y}_{n+1}\right)^{\bar{y}_{1}} \wedge \cdots \wedge \varphi\left(\bar{x}, \bar{y}_{n+1}\right)^{\bar{y}_{n}}$.

Claim 3. There are $\left(\bar{c}_{j}\right)_{j \in \omega} \in\left(M^{|B|}\right)^{\omega}$ and $\left(\bar{\gamma}_{j}\right)_{j \in \omega} \in\left(G^{n}\right)^{\omega}$ such that for all $g \in N_{A}, j \in \omega$ and $\bar{b} \in B$,

$$
\psi\left(M, \bar{\gamma}_{j}, \bar{c}_{j}\right) \cap X_{0} \subset \varphi(M, \bar{b}),
$$

$A \subset\left(\psi\left(M, \bar{\gamma}_{j+1}, \bar{c}_{j+1}\right) \cap X_{n}\right)^{g} \subset \psi\left(M, \bar{\gamma}_{j}, \bar{c}_{j}\right)$ and $\left(\psi\left(M, \bar{\gamma}_{j+1}, \bar{c}_{j+1}\right) \cap X_{n}\right)^{2} \subset \psi\left(M, \bar{\gamma}_{j}, \bar{c}_{j}\right)$.

Proof of Claim 3. We fix $\bar{b} \in B$ and first build two families $\left(\bar{c}_{j}\right)_{j \in \omega}$, $\left(\bar{\gamma}_{j}\right)_{j \in \omega}$ depending on $\bar{b}$. We take $\bar{c}_{0}=\bar{b}$ and $\gamma_{0}=(1, \ldots, 1)$. As $H$ is nice, there is $\bar{d}_{1} \in B$ such that

$$
\left(\varphi\left(M, \bar{d}_{1}\right) \cap X_{0}\right)^{2} \subset \varphi\left(M, \bar{c}_{0}\right) .
$$

By Claim 2, there are $\bar{c}_{1}$ and $\bar{\gamma}_{1}$ such that for all $g \in N_{A}$,

$$
A \subset\left(\psi\left(M, \bar{\gamma}_{1}, \bar{c}_{1}\right) \cap X_{n}\right)^{g} \subset \psi\left(M, \bar{\gamma}_{0}, \bar{d}_{1}\right) .
$$

By (6),

One also has

$$
\left(\psi\left(M, \bar{\gamma}_{1}, \bar{c}_{1}\right) \cap X_{n}\right)^{g} \subset \psi\left(M, \bar{\gamma}_{0}, \bar{d}_{1}\right) \cap X_{3} \subset \psi\left(M, \bar{\gamma}_{0}, \bar{c}_{0}\right) .
$$

$$
\left(\psi\left(M, \bar{\gamma}_{1}, \bar{c}_{1}\right) \cap X_{n}\right)^{2} \subset\left(\psi\left(M, \bar{\gamma}_{0}, \bar{d}_{1}\right) \cap X_{3}\right)^{2} \subset \psi\left(M, \bar{\gamma}_{0}, \bar{c}_{0}\right) .
$$

We go on inductively using Claim 2. As the family $\left\{\varphi(M, \bar{b}) \cap X_{0}: i \in I\right\}$ is a filter, the conclusion follows from the Compactness Theorem and the saturation assumption.

Claim 4. There is an indiscernible sequence $\left(\bar{c}_{q}\right)_{q \in \mathbf{Q}} \in\left(G^{n+1}\right)^{\omega}$ such that for all $g \in N_{A}$ rational numbers $p<q$ and $\bar{b} \in B$,

$$
\begin{gathered}
\psi\left(M, \bar{c}_{p}\right) \cap X_{0} \subset \varphi(M, \bar{b}), \\
A \subset\left(\psi\left(M, \bar{c}_{p}\right) \cap X_{n}\right)^{g} \subset \psi\left(M, \bar{c}_{q}\right) \quad \text { and }\left(\psi\left(M, \bar{c}_{p}\right) \cap X_{n}\right)^{2} \subset \psi\left(M, \bar{c}_{q}\right) .
\end{gathered}
$$

Proof of Claim 4. From Claim 3 by the Compactness Theorem and Ramsey's. 
We may now finish the proof of Theorem 5.15. For every $m>n$ define $N_{p, q}^{m} \subset X_{m}$ by

$$
N_{p, q}^{m}=\left\{g \in X_{m}:\left(X_{n}^{X_{m}} \cap \psi\left(M, \bar{c}_{p}\right)\right)^{g} \subset \psi\left(M, \bar{c}_{q}\right)\right\} .
$$

Note that $\left(X_{n}^{X_{m}}\right)_{m>n}$ is an increasing sequence so $\left(N_{p, q}^{m}\right)_{m>n}$ is decreasing. Define

$$
N=\bigcap_{m>n} \bigcap_{p<q} N_{p, q}^{m} \text { and } E=\bigcap_{q \in \mathbf{Q}} \psi\left(M, \bar{c}_{q}\right) \cap G .
$$

As $\left(X_{n}^{X_{m}}\right)^{g} \subset X_{n}^{X_{m+1}}$ for all $g \in X_{m+1}$ and $m>n$, for every rational numbers $p<r<q$ and natural number $m>n$, one has

$$
\left(N_{p, r}^{m+1} \cap N_{r, q}^{m+1}\right)^{2} \subset N_{p, q}^{m}
$$

It follows that $N \subset G$ is a subgroup. For all $m>n+1$ one has $G \subset X_{n}^{X_{m}}$ so $N$ normalises $E$. By Lemma 5.12 and indiscernibility of $\left(\bar{c}_{q}\right)_{q \in \mathbf{Q}}$, for any fixed $m>n$, there is $k(m) \in \omega$ such that every finite intersection of $N_{p, q}^{m}$ contains an intersection of at most $3 k^{2}$ sets of the form $N_{s, t}^{m+3 k}$. By the Compactness theorem, one can find a countable descending chain $N_{1} \supset N_{2} \cdots \supset N_{\ell} \supset \cdots$ of definable subsets of $X$ such that for all $\ell \geqslant 1$,

$$
N_{A} \subset N_{\ell} \subset \bigcap_{p<q} N_{p, q}^{m+\ell} \text { and } \quad N_{\ell+1}^{2} \subset N_{\ell}
$$

It follows that $\bigcap_{\ell \geqslant 1} N_{\ell}$ is a type definable subgroup of $G$ that uniformly normalises $E$. The group $E$ is nice by Remark 5.10.

5.4. External and discernible subgroups. $G$ still stands for a type definable group of type $\pi$ and base $\beta$ in the NIP structure $M$. We fix $\mathbf{M}$ a $|G|^{+}$-saturated elementary extension of $M$ and we write $\mathbf{G}$ for $\pi(\mathbf{M})$.

Definition 5.16 (external subgroup). A subgroup $H \subset G$ is external if there is a relatively definable subgroup $\mathbf{H} \subset \mathbf{G}$ (a witness) with $H=\mathbf{H} \cap G$. A family of external subgroups is uniform if there is a corresponding family of relatively definable witnesses having a common defining formula and sharing a common second base.

Lemma 5.17. The conjunction of a uniform family $\mathfrak{H}$ of external subgroups of $G$ is an external subgroup of $G$, and the family $\left\{\bigcap_{H \in \mathfrak{K}} H: \mathfrak{K} \subset \mathfrak{H}\right\}$ is uniform.

Proof. Let $\mathbf{A} \subset \mathbf{M}$ a subset and $\mathbf{X} \subset \mathbf{G}$ a definable subset such that $\varphi(\mathbf{G}, \bar{a}) \subset \mathbf{G}$ is a subgroup with second base $\mathbf{X}$ for all $\bar{a} \in \mathbf{A}$. Let $H$ be the intersection of $\varphi(G, \bar{a})$ over $\mathbf{A}$. By Lemma 5.12, we may replace the formula $\varphi(\bar{x}, \bar{y})$ by $\varphi\left(\bar{x}, \bar{y}_{1}\right) \wedge \cdots \wedge \varphi\left(\bar{x}, \bar{y}_{n}\right)$, the set $\mathbf{X}$ by an $n$th root containing $\mathbf{G}$, and assume that $\{\varphi(\mathbf{G}, \bar{a}) \cap \mathbf{X}: \bar{a} \in \mathbf{A}\}$ is a filter. The type

$$
\left.\rho(\bar{y})=\left\{(\varphi(\bar{x}, \bar{y}) \wedge \psi(\bar{x})) \subset_{\bar{x}} \varphi(\bar{x}, \bar{a})\right), \varphi(\bar{h}, \bar{y}),(\varphi(\bar{x}, \bar{y}) \wedge \psi(\bar{x}))^{2} \rightarrow \varphi(\bar{x}, \bar{y}): \bar{a} \in \mathbf{A}, \bar{h} \in H\right\}
$$

is finitely satisfiable in $\mathbf{A}$. Let $\overline{\mathbf{a}}$ be a realisation in $\mathbf{M}$. The subgroup $\varphi(\mathbf{G}, \mathbf{a}) \subset \mathbf{G}$ is relatively definable, $\mathbf{X}$ is a second base and $H=\varphi(G, \mathbf{a})$.

Definition 5.18 (discernible subgroup). A subgroup $H \subset G$ is discernible if there is a nice subgroup $\mathbf{H} \subset \mathbf{G}$ (a witness) such that $H=\mathbf{H} \cap G$. A family of discernible subgroups of $G$ is uniform if there is a corresponding family of nice witnesses that is uniform. 
Lemma 5.19. The conjunction of a uniform family $\mathfrak{H}$ of discernible subgroups of $G$ is a discernible subgroup, and the family $\left\{\bigcap_{H \in \mathfrak{K}} H: \mathfrak{K} \subset \mathfrak{H}\right\}$ is uniform.

Proof. By Lemma 5.6.

Theorem 5.20 (on external subgroups). Let $M$ be a NIP structure, $\mathbf{M} \succ M$ an $|M|^{+}$saturated elementary extension, $G=\pi(M)$ a type definable group, $\mathbf{G}=\pi(\mathbf{M})$ and $H=\mathbf{H} \cap G$ an external subgroup of $G$.

(1) There is $n \in \omega$ such that for every $A \subset G$, there are $\mathbf{a}_{1}, \ldots, \mathbf{a}_{n}$ in $\mathbf{G}$ with

$$
C_{G}(A)=C_{G}\left(\mathbf{a}_{1}, \ldots, \mathbf{a}_{n}\right) .
$$

(2) For every $n \in \omega$, there are relatively definable subgroups $\mathbf{K}, \mathbf{Z}_{n} \subset \mathbf{G}$ such that

$$
H=\mathbf{K} \cap G, \quad Z_{n}(H)=\mathbf{Z}_{n} \cap G \quad \text { and } \quad \mathbf{Z}_{n} \subset Z_{n}(\mathbf{K}) .
$$

(3) There is $n \in \omega$ such that for every $A \subset G$, there are $\mathbf{a}_{1}, \ldots, \mathbf{a}_{n}$ in $\mathbf{G}$ with

$$
H^{A}=\mathbf{H}^{\mathbf{a}_{1}} \cap \cdots \cap \mathbf{H}^{\mathbf{a}_{n}} \cap G .
$$

(4) For every $A \subset G$ containing the parameters of $\pi$, for every $\mathfrak{A} \subset$ Aut $(M / A)$, the group $H^{\mathfrak{A}}$ is external.

Proof. (1) By Example 5.8, the groups $C_{G}(a)$ form a uniform family of external subgroups. By Lemma 5.17, $C_{G}(A)$ is external, and by the proof of Lemma 5.17, $C_{G}(A)$ is of the desired form.

(2) Similar to the proof of Theorem 3.7.3 using the following claim instead of Claim 1.

Claim 5. Let $A, B \subset G$ be two subgroups, $\mathbf{D} \subset \mathbf{G}$ a relatively definable subgroup normalised by both $A$ and $B$ such that $[A, B] \subset \mathbf{D}$. Assume that $A$ and $B$ are contained in a relatively definable subgroup $\mathbf{N}_{\mathbf{D}}$ of $\mathbf{G}$ that normalises $\mathbf{D}$. There are two relatively definable subgroups $\mathbf{A}, \mathbf{B} \subset \mathbf{G}$ containing $A$ and $B$ respectively and such that $[\mathbf{A}, \mathbf{B}] \subset \mathbf{D}$.

Proof of Claim 5. Similar to the proof of Claim 1, defining

$$
\mathbf{A}(C)=\bigcap_{\substack{c \in C \\[A, c] \subset \mathbf{D}}}\left\{x \in \mathbf{N}_{\mathbf{D}}:[x, c] \subset \mathbf{D}\right\}, \quad \mathbf{B}(C)=\bigcap_{\substack{c \in C \\[c, B] \subset \mathbf{D}}}\left\{y \in \mathbf{N}_{\mathbf{D}}:[c, y] \subset \mathbf{D}\right\},
$$

(3) By Lemma 5.11, the groups $H^{a}$ form a uniform family of external subgroups. By Lemma 5.17, $H^{A}$ is external. By the proof of Lemma 5.17, $H^{A}$ is of the desired form.

(4) Let $\mathbf{M}_{1}$ be an $|M|^{+}$-homogeneous elementary extension of $\mathbf{M}$ and $\mathbf{G}_{1}=\pi\left(\mathbf{M}_{1}\right)$. For every $\sigma \in \mathfrak{A}$, let $\bar{\sigma} \in \operatorname{Aut}\left(\mathbf{M}_{1} / A\right)$ be an extension of $\sigma$ and $\overline{\mathfrak{A}}=\{\bar{\sigma}: \sigma \in \mathfrak{A}\}$. Putting $\mathbf{H}=\phi(\mathbf{G})$ and $\mathbf{H}_{1}=\phi\left(\mathbf{G}_{1}\right)$, one has $H^{\mathfrak{A}}=\mathbf{H}_{1}^{\overline{\mathfrak{A}}} \cap G$. Since $\mathbf{H}_{1}$ has a second base definable over $A$ by Example 5.7, the family $\left\{\mathbf{H}_{1}^{\bar{\sigma}}: \bar{\sigma} \in \overline{\mathfrak{A}}\right\}$ is uniform by Example 5.9. By Lemma 5.17, $H^{\mathfrak{A}}$ is external.

Theorem 5.21 (on discernible subgroups). Let $M$ be a NIP structure, $\mathbf{M} \succ M$ an $|M|^{+}$saturated elementary extension, $G=\pi(M)$ a type definable group, $\mathbf{G}=\pi(\mathbf{M})$ and $H=\mathbf{H} \cap G$ a discernible subgroup of $G$. 
(1) For every $n \in \omega$, there are nice subgroups $\mathbf{K}, \mathbf{Z}_{n} \subset \mathbf{G}$ such that

$$
H=\mathbf{K} \cap G, \quad Z_{n}(H)=\mathbf{Z}_{n} \cap G \quad \text { and } \quad \mathbf{Z}_{n} \subset Z_{n}(\mathbf{K}) .
$$

(2) For every $A \subset G$, the group $H^{A}$ is discernible.

(3) For every $A \subset G$ containing the parameters of a second base of $\mathbf{H}$ and $\pi$, for every $\mathfrak{A} \subset$ Aut $(M / A)$, the group $H^{\mathfrak{A}}$ is discernible.

(4) There are a nice subgroup $\mathbf{K} \subset \mathbf{H}$ of $\mathbf{G}$ and a type definable subgroup $\mathbf{N} \subset N_{\mathbf{G}}(\mathbf{K})$ of G such that

$$
H=\mathbf{K} \cap G \quad \text { and } \quad N_{G}(H)=\mathbf{N} \cap G .
$$

Proof. (1) Similar to the proof of Theorem 3.7.3, using the following claim instead of Claim 1.

Claim 6. Let $A, B \subset G$ two subgroups, $\mathbf{D} \subset \mathbf{G}$ a nice subgroup such that $[A, B] \subset \mathbf{D}$. Assume that $A$ and $B$ are contained in a nice subgroup $\mathbf{N}_{\mathbf{D}}$ of $\mathbf{G}$ that uniformly normalises D. There are two nice subgroups $\mathbf{A}, \mathbf{B} \subset \mathbf{G}$ containing $A$ and $B$ respectively such that $[\mathbf{A}, \mathbf{B}] \subset \mathbf{D}$.

Proof of Claim 6. As in Claim 1, there is a finite $C \subset \mathbf{N}_{\mathbf{D}}$ such that, defining for any $S \subset \mathbf{N}_{\mathbf{D}}$

$$
\mathbf{A}(S)=\bigcap_{\substack{c \in S \\[A, c] \subset \mathbf{D}}}\left\{x \in \mathbf{N}_{\mathbf{D}}:[x, c] \subset \mathbf{D}\right\} \quad \text { and } \quad \mathbf{B}(S)=\bigcap_{\substack{c \in S \\[c, B] \subset \mathbf{D}}}\left\{y \in \mathbf{N}_{\mathbf{D}}:[c, y] \subset \mathbf{D}\right\},
$$

one has $[\mathbf{A}(A \cup B \cup C), \mathbf{B}(A \cup B \cup C)] \subset \mathbf{D}$. Let $\left\{\varphi\left(x, a_{i}\right): i \in I\right\}$ be the type that defines $\mathbf{D}$ relatively to $\mathbf{G}$ and let $\beta_{2}=\phi_{2}(M)$. Let us show that, for any $S \subset \mathbf{N}_{\mathbf{D}}$, putting

$$
\mathbf{H}_{i}(s)=\left\{g \in \phi_{2}(\mathbf{M}):[g, s] \subset \varphi\left(\mathbf{M}, a_{i}\right)\right\},
$$

the family $\mathfrak{H}=\left\{\bigcap_{i \in I} \mathbf{N}_{\mathbf{D}} \cap \mathbf{H}_{i}(s): s \in S\right\}$ is a uniform family of nice subgroups in $\mathbf{N}_{\mathbf{D}}$. By Lemma 5.6, this will show that $\mathbf{A}(A \cup B \cup C)$ and $\mathbf{B}(A \cup B \cup C)$ are nice in $\mathbf{N}_{\mathbf{D}}$, hence in $\mathbf{G}$. We need only find a common second base for the members of $\mathfrak{H}$. As $\mathbf{D}$ is nice in $\mathbf{G}$, there is a definable set $\mathbf{Y}$ with $\mathbf{G} \subset \mathbf{Y}$ such that for all $i \in I$ there is $j \in I$ with

$$
\left(\mathbf{Y} \cap \varphi\left(\mathbf{M}, a_{j}\right)\right)^{2} \subset \varphi\left(\mathbf{M}, a_{i}\right) .
$$

As $\mathbf{D}$ is uniformly normal in $\mathbf{N}_{\mathbf{D}}$, there is a definable $\mathbf{Z}$ with $\mathbf{N}_{\mathbf{D}} \subset \mathbf{Z} \subset \phi_{2}(\mathbf{M})$ such that for all $j \in I$, there is $k \in I$ such that for all $g \in \mathbf{Z}$,

$$
\left(\mathbf{Z} \cap \varphi\left(\mathbf{M}, a_{k}\right)\right)^{g} \subset \varphi\left(\mathbf{M}, a_{j}\right) .
$$

There are also definable sets $\mathbf{Y}^{1 / 6}$ and $\mathbf{Z}^{1 / 4}$ containing $\mathbf{N}_{\mathbf{D}}$ with

$$
\left(\mathbf{Y}^{1 / 6}\right)^{6} \subset \mathbf{Y} \quad \text { and } \quad\left(\mathbf{Z}^{1 / 4}\right)^{4} \subset \mathbf{Z}
$$

We put $\mathbf{W}=\phi_{3}(\mathbf{M}) \cap \mathbf{Y}^{1 / 6} \cap \mathbf{Z}^{1 / 4}$ and claim that $\left(\mathbf{W} \cap \mathbf{H}_{k}(s)\right)^{2} \subset \mathbf{H}_{i}(s)$ for any $s \in \mathbf{N}_{\mathbf{D}}$. For all elements $g, h \in \mathbf{W} \cap \mathbf{H}_{k}(s)$, one has

$$
[g, s]^{h} \in\left(\left(\mathbf{Z}^{1 / 4}\right)^{4} \cap \varphi\left(\mathbf{M}, a_{k}\right)\right)^{h} \quad \text { and } \quad[h, s] \in\left(\mathbf{Z}^{1 / 4}\right)^{4} \cap \varphi\left(\mathbf{M}, a_{k}\right),
$$

and so

$$
[g, s]^{h} \in \varphi\left(\mathbf{M}, a_{j}\right) \quad \text { and } \quad[h, s] \in \varphi\left(\mathbf{M}, a_{j}\right)
$$


hence

$$
[g h, s]=[g, s]^{h}[h, s] \in\left(\left(\mathbf{Y}^{1 / 6}\right)^{6} \cap \varphi\left(\mathbf{M}, a_{j}\right)\right)^{2} \subset \varphi\left(\mathbf{M}, a_{i}\right) .
$$

This shows that $g h$ belongs to $\mathbf{H}_{i}(s)$ and that $\mathbf{W}$ is the desired second base.

(2) $H^{A}$ is discernible by Lemma 5.11 and Lemma 5.19 .

(3) Let $\mathbf{M}_{1}$ be an $|M|^{+}$-homogeneous elementary extension of $\mathbf{M}$ and $\mathbf{G}_{1}=\pi\left(\mathbf{M}_{1}\right)$. For every $\sigma \in \mathfrak{A}$, let $\bar{\sigma} \in \operatorname{Aut}\left(\mathbf{M}_{1} / A\right)$ be an extension of $\sigma$ and $\overline{\mathfrak{A}}=\{\bar{\sigma}: \sigma \in \mathfrak{A}\}$. Putting $\mathbf{H}=\phi(\mathbf{G})$ and $\mathbf{H}_{1}=\phi\left(\mathbf{G}_{1}\right)$, one has $H^{\mathfrak{A}}=\mathbf{H}_{1}^{\overline{\mathfrak{A}}} \cap G$, so $H^{\mathfrak{A}}$ is discernible by Example 5.9 and Lemma 5.19.

(4) By Theorem 5.15.

Corollary 5.22. Let $G$ be a NIP group, $A \subset G$ a subset and $n \in \omega$, then $C_{G}^{n}(A)$ is discernible.

Proof. The $n$th centraliser of $A$ is defined by induction on $n$ putting $C_{G}^{0}(A)=\{1\}$ and

$$
C_{G}^{n+1}(A)=\bigcap_{k \leqslant n} N_{G}\left(C_{G}^{k}(A)\right) \cap\left\{g \in G:[g, A] \subset C_{G}^{n}(A)\right\} .
$$

We proceed by induction on $n$. For $n=1$, this is Theorem 3.7.1. Assume that $C_{G}^{n}(A)=$ $\mathbf{C}_{n} \cap G$ where $\mathbf{C}_{n} \subset \mathbf{G}$ is nice. One has $\left[C_{G}^{n+1}(A), A\right] \subset \mathbf{C}_{n}$. As $A$ and $C_{G}^{n+1}(A)$ normalise $C_{G}^{n}(A)$, by Theorem 3.8.3 applied to $\mathbf{C}_{n} \cap G$, there is a nice subgroup $\mathbf{N} \subset \mathbf{G}$ that normalises $\mathbf{C}_{n}$ (hence normalise it uniformly) and contains both $A$ and $C_{G}^{n+1}(A)$. By Claim 6 , there is a nice subgroup $\mathbf{H} \subset \mathbf{G}$ such that $[\mathbf{H}, A] \subset \mathbf{C}_{n}$ and $C_{G}^{n+1}(A) \subset \mathbf{H}$. By induction hypothesis and Theorem 3.8.3, there is a nice subgroup $\mathbf{M}$ of $G$ such that

$$
\mathbf{M} \cap G=\bigcap_{k \leqslant n} N_{G}\left(C_{G}^{k}(A)\right)
$$

Putting $\mathbf{C}_{n+1}=\mathbf{M} \cap \mathbf{H}$, one has

$$
C_{G}^{n+1}(A)=\mathbf{C}_{n+1} \cap G .
$$

\section{ENVELOPES IN TYPE DEFInABLE GROUPS}

Let $M$ be a NIP structure and $G$ a type definable group. We fix a $|G|^{+}$-saturated extension $\mathbf{M}$ of $M$ and write $\mathbf{G}$ for $\pi(\mathbf{M})$.

Theorem 6.1 (abelian envelope). Let $A \subset G$ be an abelian subgroup. There is an external subgroup $H=\mathbf{H} \cap G$ with $A \subset H$ such that $\mathbf{H}$ is abelian, $H$ is $A$-invariant and normalised by $N_{G}(A)$.

Proof. The $A$-invariant subgroup $Z\left(C_{G}(A)\right)$ contains $A$ and is normalised by $N_{G}(A)$. It is an external abelian subgroup by Theorem 5.20.1 and 5.20.2.

Theorem 6.2 (nilpotent envelope). Let $N \subset G$ be a nilpotent subgroup of class $n$. There is an external subgroup $H=\mathbf{H} \cap G$ with $N \subset H$ such that $\mathbf{H}$ is nilpotent of class $n, H$ is $N$-invariant and normalised by $N_{G}(N)$. 
Proof. By induction on $k \leqslant n$, we build a chain $\mathbf{Z}_{0} \triangleleft \cdots \triangleleft \mathbf{Z}_{k}$ of relatively definable subgroups of $\mathbf{G}$ such that

$$
\mathbf{Z}_{0}=\{1\}, \quad Z_{k}(N) \subset \mathbf{Z}_{k} \quad \text { and } \quad\left[\mathbf{Z}_{k}, N\right] \subset \mathbf{Z}_{k-1} .
$$

If $\mathbf{Z}_{k}$ is built, as one has

$$
\left[Z_{k+1}(N), N\right] \subset Z_{k}(N) \subset \mathbf{Z}_{k}
$$

and as $N$ is contained in the subgroup $C_{\mathbf{G}}\left(\mathbf{Z}_{k} / \mathbf{Z}_{k-1}\right)$ which normalises $\mathbf{Z}_{k}$, by Claim 5 , there is a relatively definable subgroup $\mathbf{Z}_{k+1}$ of $\mathbf{G}$ containing $Z_{k+1}(N)$ such that

$$
\left[\mathbf{Z}_{k+1}, N\right] \subset \mathbf{Z}_{k}
$$

For every $k \leqslant n$, the group

$$
\mathbf{H}_{k}=\left\{x \in \mathbf{Z}_{n}:\left[\mathbf{Z}_{k}, x\right] \subset \mathbf{Z}_{k-1}\right\}
$$

is external by Lemma 5.17, and the group $\mathbf{H}_{1} \cap \cdots \cap \mathbf{H}_{n}$ is a nilpotent group of class $n$ that contains $N$. This finishes the induction, and one concludes with Theorem 5.20.3 and 5.20.4.

Theorem 6.3 (soluble envelope). Let $S \subset G$ be a soluble subgroup of derived length $\ell$. There is a type definable subgroup $\mathbf{N} \subset \mathbf{G}$ with $N_{G}(S) \subset \mathbf{N}$ and a nice and uniformly normal subgroup $\mathbf{H} \subset \mathbf{N}$ that contains $S$ and is soluble of derived length $\ell$. More precisely, $\mathbf{H}$ is the intersection of a uniform family of relatively definable subgroups of $\mathbf{N}$.

Proof. Let us consider the derived series $S \triangleright S^{(1)} \triangleright \cdots \triangleright S^{(\ell)}$, and let $\mathbf{H}_{1}$ be a relatively definable abelian subgroup of $\mathbf{G}$ that contains $S^{(\ell-1)}$ and that is normalised by $N_{G}(S)$. Let $\mathbf{N}_{1}$ be a type definable subgroup of $\mathbf{G}$ that normalises $\mathbf{H}_{1}$ and contains $N_{G}(S)$. We build by induction on $k \leqslant \ell$ two families $1 \triangleleft \mathbf{H}_{1} \triangleleft \cdots \triangleleft \mathbf{H}_{k}$ and $\mathbf{N}_{1}, \ldots, \mathbf{N}_{k}$ of subgroups of $\mathbf{G}$ such that for every $k \leqslant \ell$, the group $\mathbf{N}_{k}$ is a type definable subgroup of $\mathbf{G}$ that contains $N_{G}(S)$ and $\mathbf{H}_{k}$ is a nice and uniformly normal subgroup of $\mathbf{N}_{k}$ that satisfies

$$
S^{(\ell-k)} \subset \mathbf{H}_{k} \quad \text { and } \quad\left[\mathbf{H}_{k}, \mathbf{H}_{k}\right] \subset \mathbf{H}_{k-1} .
$$

If $\mathbf{N}_{k}$ and $\mathbf{H}_{k}$ are built, one has

$$
\left[S^{(\ell-k-1)}, S^{(\ell-k-1)}\right] \subset S^{(\ell-k)} \subset \mathbf{H}_{k}
$$

By Claim 6, there is a nice subgroup $\mathbf{K}_{k+1}$ of $\mathbf{N}_{k}$ such that

$$
S^{(\ell-k-1)} \subset \mathbf{K}_{k+1} \quad \text { and } \quad\left[\mathbf{K}_{k+1}, \mathbf{K}_{k+1}\right] \subset \mathbf{H}_{k} .
$$

By Theorem 5.21.4 there is a type definable subgroup $\mathbf{N}_{k+1}$ of $\mathbf{G}$ that contains $N_{G}(S)$ and a nice subgroup $\mathbf{H}_{k+1}$ of $\mathbf{K}_{k+1}$ that contains $S^{(\ell-k-1)}$ and is uniformly normalised by $\mathbf{N}_{k+1}$. We put

$$
\mathbf{N}=\mathbf{N}_{1} \cap \cdots \cap \mathbf{N}_{\ell} \quad \text { and } \quad \mathbf{H}=\mathbf{H}_{\ell}
$$




\section{REFERENCES}

[AB14] Tuna Altmel and Paul Baginski, Definable envelopes of nilpotent subgroups of groups with chain conditions on centralizers, Proc. Amer. Math. Soc. 142 (2014), no. 5, 1497-1506.

[Adl14] Hans Adler, An introduction to theories without the independence property, 2014.

[Bai01] Bektur Sembiuly Baizhanov, Expansion of a model of a weakly o-minimal theory by a family of unary predicates, J. Symbolic Logic 66 (2001), no. 3, 1382-1414.

[Bél12] Luc Bélair, Panorama of p-adic model theory, Ann. Sci. Math. Québec 36 (2012), no. 1, 43-75 (2013).

[BS76] John T. Baldwin and Jan Saxl, Logical stability in group theory, J. Austral. Math. Soc. Ser. A 21 (1976), no. 3, 267-276.

[CP12] Annalisa Conversano and Anand Pillay, Connected components of definable groups and o-minimality I, Adv. Math. 231 (2012), no. 2, 605-623.

[dA13] Ricardo de Aldama, Definable nilpotent and soluble envelopes in groups without the independence property, MLQ Math. Log. Q. 59 (2013), no. 3, 201-205.

[Del81] Françoise Delon, Types sur $\mathbf{C}((X))$, Study Group on Stable Theories (Bruno Poizat), Second year: 1978/79 (French), Secrétariat Math., Paris, 1981, pp. Exp. No. 5, 29.

[Del89] — Définissabilité avec paramètres extérieurs dans $\mathbf{Q}_{p}$ et $\mathbf{R}$, Proc. Amer. Math. Soc. 106 (1989), no. 1, 193-198.

[Fis77] Edward R. Fisher, Abelian structures. I, Abelian group theory (Proc. Second New Mexico State Univ. Conf., Las Cruces, N.M., 1976), Springer, Berlin, 1977, pp. 270-322. Lecture Notes in Math., Vol. 616.

[GS84] Yuri Gurevich and Peter H. Schmitt, The theory of ordered abelian groups does not have the independence property, Trans. Amer. Math. Soc. 284 (1984), no. 1, 171-182.

[HH84] Victor Harnik and Leo Harrington, Fundamentals of forking, Ann. Pure Appl. Logic 26 (1984), no. 3, 245-286.

[HPP11] Ehud Hrushovski, Ya'acov Peterzil, and Anand Pillay, On central extensions and definably compact groups in o-minimal structures, J. Algebra 327 (2011), 71-106.

[KSW11] Itay Kaplan, Thomas Scanlon, and Frank O. Wagner, Artin-Schreier extensions in NIP and simple fields, Israel J. Math. 185 (2011), 141-153.

[Las92] Michael C. Laskowski, Vapnik-Chervonenkis classes of definable sets, J. London Math. Soc. (2) 45 (1992), no. 2, 377-384.

[LL08] Michel L. Lapidus and Hùng Lũ, Nonarchimedean Cantor set and string, J. Fixed Point Theory Appl. 3 (2008), no. 1, 181-190.

[Mat93] Larry Matthews, The independence property in unstable algebraic structures I: p-adically closed fields, 1993.

[MS08] Dugald Macpherson and Charles Steinhorn, One-dimensional asymptotic classes of finite structures, Trans. Amer. Math. Soc. 360 (2008), no. 1, 411-448 (electronic).

[MT12] Dugald Macpherson and Katrin Tent, Pseudofinite groups with NIP theory and definability in finite simple groups, Groups and model theory, Contemp. Math., vol. 576, Amer. Math. Soc., Providence, RI, 2012, pp. 255-267.

[Neu54] B. H. Neumann, Groups covered by permutable subsets, J. London Math. Soc. 29 (1954), 236-248.

[Pil88] Anand Pillay, On groups and fields definable in o-minimal structures, J. Pure Appl. Algebra 53 (1988), no. 3, 239-255.

[Poi85] Bruno Poizat, Cours de théorie des modèles, Bruno Poizat, Lyon, 1985, Une introduction à la logique mathématique contemporaine. [An introduction to contemporary mathematical logic].

[Poi87] _ Groupes stables, Nur al-Mantiq wal-Ma’rifah [Light of Logic and Knowledge], 2, Bruno Poizat, Lyon, 1987, Une tentative de conciliation entre la géométrie algébrique et la logique mathématique. [An attempt at reconciling algebraic geometry and mathematical logic]. 
[She90] Saharon Shelah, Classification theory and the number of nonisomorphic models, second ed., Studies in Logic and the Foundations of Mathematics, vol. 92, North-Holland Publishing Co., Amsterdam, 1990 .

[She09] _ Dependent first order theories, continued, Israel J. Math. 173 (2009), 1-60.

[Szm55] Wanda Szmielew, Elementary properties of Abelian groups, Fund. Math. 41 (1955), 203-271.

[VC71] Vladimir N. Vapnik and Alexey Ya. Chervonenkis, On the uniform convergence of relative frequencies of events to their probabilities., Theory Probab. Appl. 16 (1971), 264-280 (English).

[vdD86] Lou van den Dries, Tarski's problem and Pfaffian functions, Logic colloquium '84 (Manchester, 1984), Stud. Logic Found. Math., vol. 120, North-Holland, Amsterdam, 1986, pp. 59-90.

[Wag97] Frank O. Wagner, Stable groups, London Mathematical Society Lecture Note Series, vol. 240, Cambridge University Press, Cambridge, 1997.

[Wei93] Volker Weispfenning, Quantifier elimination for abelian structures, 1993.

[Woo79] Carol Wood, Notes on the stability of separably closed fields, J. Symbolic Logic 44 (1979), no. 3, $412-416$.

Université GaLATASARAY

FACUlté de SCIEnCES ET De LetTres

DÉpartement de Mathématiques

ÇIRAĞAN CADDESI N ${ }^{\circ} 36$

34357 Ortaköy, İstanbul, TÜrkiye

UNIVERSITÄT KONSTANZ

FaChBereich Matematik und Statistik

78457 Konstanz, Germany

(current address) PÔle De Mathématiques DE L'INSA DE LyON, Bâtiment Léonard de Vinci - 21, aVenue Jean Capelle, 69621 Villeurbanne, France

E-mail address: cedric.milliet@insa-lyon.fr 